\title{
Sequence conservation and structural features that are common within TRP channels
}

Deny Cabezas-Bratesco ${ }^{1, \&}$, Charlotte K. Colenso ${ }^{1,2, \&}$, Katina Zavala ${ }^{3}$, Danielle Granata ${ }^{4}$, Vincenzo Carnevale $^{4}$, Juan C. Opazo ${ }^{3,5,6}$, Sebastian E. Brauchi ${ }^{1,6,7}$ iscep?

${ }^{1}$ Instituto de Fisiologia, Facultad de Medicina, Universidad Austral de Chile, Valdivia, 511-0566, Chile.

${ }^{2}$ School of Cellular and Molecular Medicine, University of Bristol, Bristol, BS8 1TD, UK.

${ }^{3}$ Instituto de Ciencias Ambientales y Evolutivas, Facultad de Ciencias, Universidad Austral de Chile, Valdivia, Chile.

${ }^{4}$ Institute for Computational Molecular Science, Temple University, Philadelphia, USA.

${ }^{5}$ Integrative Biology Group, Universidad Austral de Chile, Valdivia, Chile

${ }^{6}$ Millennium Nucleus of Ion Channel-associated Diseases (MiNICAD), Valdivia, Chile.

7Janelia Research Campus, Howard Hughes Medical Institute, Ashburn, VA, 20147, USA.

\&D.C-B. and C.K.C. equally contributed to this work.

Correspondence should be addressed to Dr. Sebastian Brauchi: sbrauchi@uach.cl

Running title: A Structural Signature in TRP Channels. 


\section{ABSTRACT}

2 TRP proteins are a large family of cation selective channels, surpassed in variety only by

3 voltage-gated potassium channels. Detailed molecular mechanisms governing how

4 membrane voltage, ligand binding, or temperature can induce conformational changes

5 promoting the open state of the channel are still missing for TRP channels. Aiming to unveil

6 distinctive structural features common to the transmembrane domains within the TRP

7 family, we performed bioinformatic analyses over a large set of TRP channel genes. Here

8 we report a discrete and exceptionally conserved set of residues. This fingerprint is

9 composed of eleven residues localized at equivalent three-dimensional positions in TRP

10 channels from the different subtypes. Moreover, these amino acids are arranged in three

11 groups, connected by a set of aromatics located at the core of the transmembrane structure.

12 We hypothesize that differences in the connectivity between these different groups of

13 residues harbors the apparent differences in coupling strategies used by TRP subgroups. 
21 Transient receptor potential (TRP) proteins constitute a large family of cation-selective ion

22 channels involved in a number of physiological functions (Clapham, 2003; Nilius et al.,

23 2007). TRPs have been related to all voltage-gated cation channels (VGCC), two-pore

24 sodium channels (TPCNs), and CatSper channels (Yu et al., 2005). The TRP family in

25 vertebrates is composed by of 28 channels, belonging to two major groups and seven

26 subfamilies or subtypes: TRPA1, TRPV, TRPC, TRPM, TRPN, PKD2s, and TRPML (Ramsey et

27 al., 2006). While group I gathers members of TRPC, TRPM, TRPV, TRPA, and TRPN

28 subfamilies, group II is composed of members of the TRPML and PKD2 channels

29 (Venkatachalam 2007, Himmel 2020). In general, TRP channels share poor cation

30 selectivity and a loose sequence similarity (Ramsey et al., 2006). Cataloged as polymodal

31 ion channels, they have the ability to integrate multiple stimuli (e.g., chemical, mechanical,

32 electrical, and thermal) to promote channel opening. Such polymodality observed in TRPs

33 has been explained in terms of allosteric interactions (Brauchi et al., 2004; Latorre et al.,

34 2009). Different lines of research have shown that sensor modules -activated by specific

35 mechanisms- couple to each other and to the channel pore, modulating permeation (Hui et

36 al., 2003; Castillo et al., 2018; Zubcevic et al., 2019; Zhao et al., 2020; Yang et al., 2018; Yang

37 et al., 2020).

38 Structural data revealed that TRP channels share the general architecture of

39 VGCCs (Kasimova et al., 2016; Cheng, 2018; Cao, 2020). They assemble as domain-swapped

40 homotetramers, with monomers containing six transmembrane segments (i.e., TM1-TM6) 
41 flanked by cytoplasmic N- and C-terminal domains. The transmembrane helices TM5 and

42 TM6, together with the section between them, give shape to the conductive pore (Ramsey

43 et al., 2006; Liao et al., 2013). The first four transmembrane helices and part of the

44 intracellular domains have been described as regulatory regions as they provide binding

45 sites for agonists and cofactors (Steinberg et al., 2014; Voolstra \& Huber, 2014; Cao, 2020).

46 The transmembrane region between TM1 and TM4 shows fundamental differences with

47 VGCCs, where the absence of charged residues within the transmembrane region should be

48 underscored (Palovcak et al., 2015; Cao, 2020). This could explain the modest voltage

49 dependence observed in TRP channels (TRPMs and TRPVs are tenfold less voltage

50 dependent compared to VGCCs) that might be supported by residues located in the pore

51 region (Liu et al., 2009; Yang et al., 2020). The transmembrane region between TM1 and

52 TM4 hosts binding pockets for ligands in all the different TRP channel subtypes, serving as

53 a ligand-binding domain (LBD) in TRPs (Steinberg et al., 2014; Huffer et al., 2020).

54 Nevertheless, this LBD has been historically referred to as the voltage-sensing like domain

55 (VSLD).

56 Regardless of the considerable variability in sequence similarity and physiological function,

57 it is clear that TRP channels are closely related, specially those that belongs to group

58 I (Kadowaki, 2015; Peng et al., 2015; Arias-Darraz et al., 2015). The structural

59 understanding of TRP proteins has been strengthened by technical advances allowing the

60 recent release of a large set of high-resolution cryo-electron microscopy (cryo-EM)

61 structures (Cao, 2020; Samanta et al., 2019). Led by the structure of TRPV1, nowadays 
62 structures are available for at least one member of each subfamily, including crTRP1 from

63 the green algae Chlamydomonas reinhardtii (McGoldrick et al., 2019; Cao, 2020). Together

64 with advances in structural biology, intense research during recent years has been focused

65 into understanding the molecular mechanisms supporting TRP activation and

66 regulation (Hofmann et al., 2017; Yang \& Zheng, 2017; Yang et al., 2018; Singh, et al., 2018;

67 Hilton et al., 2019; Zhang et al., 2019; Zubcevic et al., 2019; Zhao et al., 2020, Nadezhdin et

68 al., 2021; Nadezhdin et al., 2021a). Although a consistent picture accounting for general

69 principles governing TRP channels' mechanics is still missing, several structural features

70 have been identified of great importance. Among these the $\mathrm{N}$ - and C-terminal domains

71 flanking the transmembrane region namely the preTM1 and the TRP domain helix (TDh)

72 respectively. These elements, seemingly related to the integration of molecular mechanics

73 during activation, are present in all members of the group I of TRPs and absent in TRP

74 channels from group II (McGoldrick et al., 2019; Zhao et al., 2020; Nadezhdin et al., 2021).

75 Here we studied TRP channel proteins from a large variety of organisms aiming to find

76 conserved amino acid motifs. We found a discrete number of highly conserved residues in

77 all TRP channels from group I (GI-TRPs). Moreover, strong patterns of conservation were

78 found unique to the different TRP subtypes. The amino acid conservation can be traced

79 down to TRP channels from unicellular organisms, suggesting a robust architectural design.

80 In addition, we identified a group of aromatic residues facing the core of the LBD (i.e. TM 1-

814 ) in all subtypes. In agreement with our phylogenetic reconstruction this aromatic core

82 can be traced down to crTRP1, it is absent in VGCCs, and present in a rudimentary form in 
83 TPCNs. Further analyses of the highly conserved residues unveiled inter-subunit

84 interactions connecting the aromatic core of one subunit with signature residues located at

85 the selectivity filter of the neighboring subunit. Overall, our results suggest that TRP

86 channel specialization has been built around the connectivity of heavily conserved distant

87 residues that are located at critical sites within the structure.

\section{RESULTS}

\section{Phylogenetic relationships of GI-TRPs.}

91 We first investigated the phylogenetic position of the unicellular TRP channels included in

92 this study. To this end we reconstructed a phylogenetic tree including TRPs from amniotes

93 in addition to unicellular sequences we previously identified as TRP channels (Fig. 1)

94 (Arias-Darraz et al., 2015). The topology of the phylogenetic reconstruction is in agreement

95 with previous reports (Himmel et al., 2020). According to our phylogenetic tree, we

96 recovered TRP channels from unicellular organisms into five clades (UC1-5, Fig. 1). A clade

97 containing TRP sequences from Dictyostelium discoideum, Dictyostelium purpureum and

98 Paramecium tetraurelia was recovered sister to the clade including TRPVs and TRPA1

99 sequences (UC3, Fig. 1). In turn, a group including sequences from Dictyostelium

100 discoideum, Dictyostelium purpureum and Coccomyxa subellipsoidea was recovered sister to

101 the UC3/ TRPVs/TRPA1 clade (UC2, Fig. 1). A well supported clade containing five 102 sequences from Chlamydomonas reinhardtii, one sequence from Leishmania mexicana, two

103 sequences from Micromonas pusilla and a single sequence from Volvox carteri was 
bioRxiv preprint doi: https://doi.org/10.1101/209CB.06.459056; this version posted September 6, 2021. The copyright holder for this preprint (which was not certified by peer review althor/funder whe has granted bioRxiv a license to display the preprint in perpetuity. It is made

Figure 1. Maximum likelihood tree showing relationships among Group I of TRP channels from amniotes and the putative TRPs from unicellular organisms. The scale denotes substitutions per site and colors represent lineages. Human (Homo sapiens) and chicken (Gallus gallus) Potassium voltage-gated channel subfamily A member 2 (KCNA2), and Sodium Voltage-Gated Channel Alpha Subunit 8 (SCN8A) sequences were included as an outgroup. 
104 recovered sister to the above mentioned clade (UC1, Fig. 1). Thus, this branching pattern

105 suggests that these three unicellular clades are more related to TRPVs and TRPA1 channels

106 than to any other channel in the TRP gene family. We also recovered a single sequence from

107 Chlamydomonas reinhardtii (i.e. crTRP1) sister to the TRPC clade with strong support (UC5,

108 Fig. 1), suggesting that this sequence from this single-cell green algae shares a common

109 ancestor more recently in time with TRPCs than with any other gene family member.

110 Finally, we recovered a clade containing sequences from Leishmania infantum, Leishmania

111 major, Leishmania mexicana, Trypnosoma cruzi and Paramecium tetraurelia sister to all

112 other TRP sequences included in our study (UC4, Fig. 1).

113

\section{Multiple sequence alignments identify a discrete set of highly conserved residues}

115 We then focused our attention on the transmembrane regions and the immediate flanking

116 segments that are common to all TRPs. In particular, the segment containing the last

117 portion of the pre-TM1 region, the transmembrane region, and the TRP domain helix

118 (TDh). We then constructed a multiple sequence alignment using TRPs included in our

119 phylogenetic analysis (including those from UC groups 1-5) and a set of non-redundant

120 TRP channel sequences gathered from the UniProt database (Figure 2a). In agreement with

121 previous observations (Huffer et al., 2020), gaps are in general confined to extracellular

122 loops and almost nonexistent at the intracellular linker between TM4-TM5 and the TDh

123 (Figure 2a). Moreover, the pattern of these gaps in the extracellular loops seems to be a

124 good predictor for the subfamily grouping. This is the case of the pore loop region, which is 


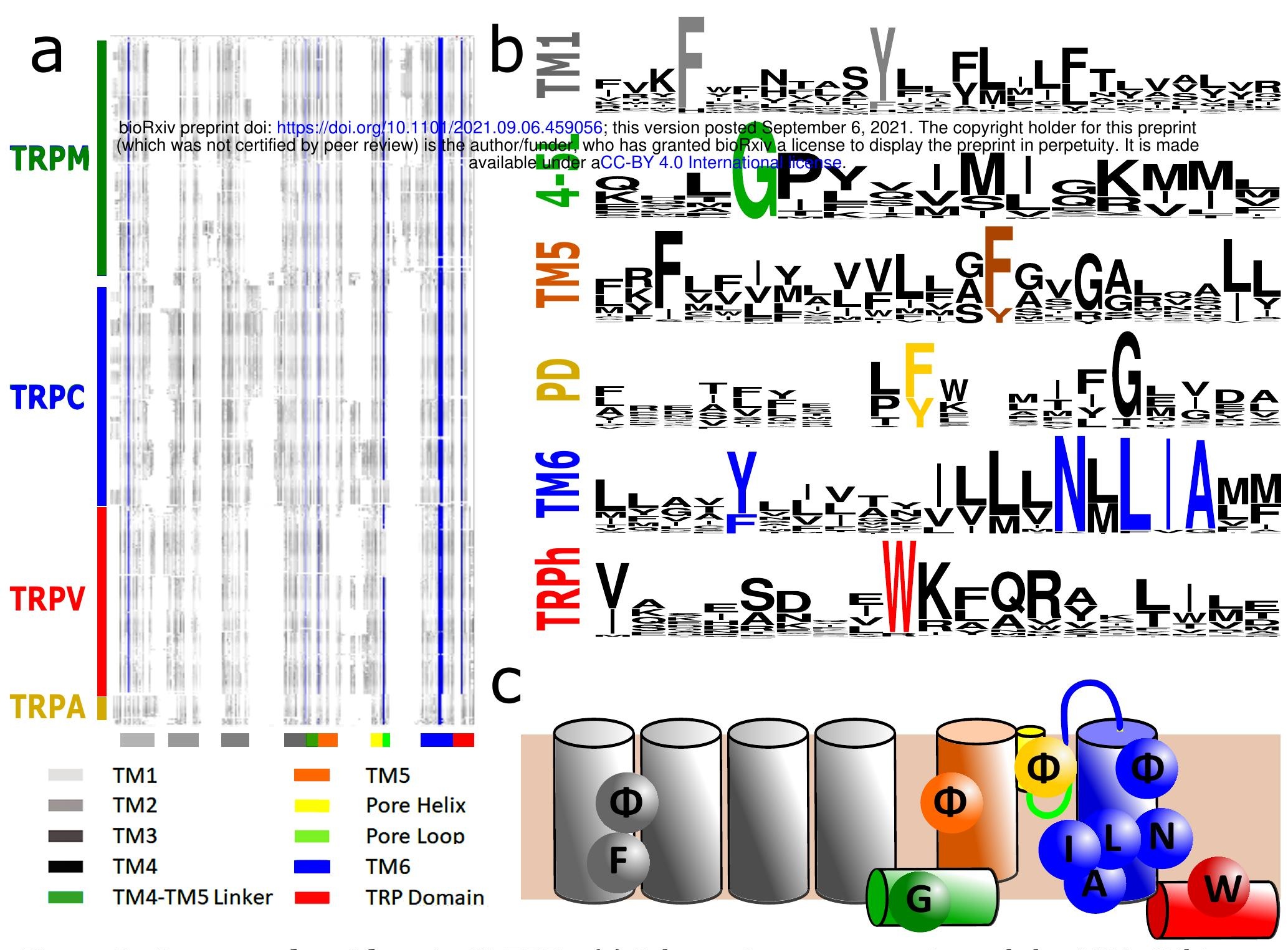

Figure 2. Conserved residues in CI-TRPs. (a) Schematic representation of the MSA. White blanks correspond to gaps in the alignment. Gray shades represent nonconserved residues. Blue shades correspond to sequence identities ( $>90 \%)$. TRP channel subfamilies are indicated on the left. Structural mapping is indicated below in color code. (b) Sequence logos for the TRP family, depicting highly conserved residues (>90\% identity). (c) Cartoon of a TRP channel monomer depicting the location of conserved residues in the secondary structure. $\Phi$ denotes 6 carbon aromatic residues (i.e., Tyr or Phe). 
125 comparatively longer in TRPM channels, or the extended TM3-TM4 loop seen in channels

126 from the TRPC family (Figure 2a). This observation holds true for the individual alignment

127 of each subfamily, where a correlation between the pattern of the gaps and the different

128 members within subfamilies is evident (Supplementary Figure 1). We tested the usefulness

129 of these patterns as predictors by looking at Drosophila channels TRPL and TRPgamma that

130 were included in the dataset pulled from Uniprot. We observed that TRPL and TRPgamma

131 segregate to TRPCs and within the subtype, they showed a unique pattern of sequence

132 similarity and gaps -similar to a bar code- allowing their identification from other

133 subfamily members (Supplementary Figure 1).

134 A thorough analysis of the distribution of amino acids identified a discrete set of highly

135 conserved residues (identity $>90 \%$ ) common to the TRP superfamily (Figure 2; Table 1).

$13670 \%$ of TRP channels analyzed have 11 conserved and non-contiguous amino acids we

137 interpreted as an amino acid signature or fingerprint (F $\boldsymbol{\Phi}$ G $\boldsymbol{\Phi} \boldsymbol{\Phi} \boldsymbol{\Phi} \mathbf{N} \mathbf{L} \mathbf{I} \mathbf{A} \mathbf{W}$, where $\Phi$

138 could be either Phe or Tyr; Figure 2b). We observed that 95\% of the TRP channels

139 contained (1311 sequences in total) at least 8 of these conserved side chains. In contrast,

140 the bacterial potassium channel KvAP shows five of these residues while Kv1.2 exhibits

141 only three (Table 1).

142 This fingerprint was used to compare the conservation between TRP channel subtypes.

143 While TRPCs and TRPVs conserve the complete set of 11 residues (F $\boldsymbol{\Phi} \mathbf{G} \boldsymbol{\Phi} \mathbf{F} \boldsymbol{\Phi} \mathbf{N} \mathbf{L} \mathbf{I} \mathbf{A} \mathbf{W}$ ),

144 TRPMs and TRPNs show 10 (F Y G $\mathbf{\Phi}$ Y $\mathbf{\Phi} \mathbf{N} \mathbf{L}$ V A W), and TRPA1 only 8 (H Y G F F F N L I G

$145 \mathrm{R})$. The differences observed in TRPA1 can be mapped to the first turn of the TM1 helix and 


\begin{tabular}{|c|c|c|c|c|c|c|c|c|c|c|c|c|}
\hline Sign & $\mathbf{F}$ & $\Phi$ & $\mathbf{G}$ & $\Phi$ & $\Phi$ & $\Phi$ & $\mathbf{N}$ & $\mathbf{L}$ & $\mathbf{I}$ & A & $\mathbf{W}$ & \\
\hline$\%$ Ident & 92.0 & 90.4 & 98.6 & 97.4 & 97.2 & 98.7 & 95.0 & 95.4 & 90.5 & 94.5 & 92.5 & \\
\hline Pos TRPV1 & 434 & 441 & 563 & 591 & 638 & 666 & 676 & 678 & 679 & 680 & 697 & Ids \\
\hline TRPVs & $\mathbf{F}$ & $\Phi$ & G & $\Phi$ & $\mathbf{F}$ & $\Phi$ & $\mathbf{N}$ & $\mathbf{L}$ & I & A & w & 11 \\
\hline TRPMS & $\mathbf{F}$ & $\mathbf{Y}$ & G & $\Phi$ & $\mathbf{Y}$ & $\Phi$ & $\mathbf{N}$ & $\mathbf{L}$ & V & A & W & 10 \\
\hline TRPCS & $\mathbf{F}$ & $\Phi$ & G & $\mathbf{F}$ & $\mathbf{F}$ & $\Phi$ & $\mathbf{N}$ & $\mathbf{L}$ & I & A & W & 11 \\
\hline TRPN1 & $\mathbf{F}$ & H & G & $\mathbf{F}$ & $\mathbf{F}$ & $\mathbf{Y}$ & $\mathbf{N}$ & $\mathbf{L}$ & I & A & W & 10 \\
\hline TRPA1 & H & $\mathbf{Y}$ & G & $F$ & $\mathbf{F}$ & $\mathbf{F}$ & $\mathbf{N}$ & $\mathbf{L}$ & I & G & $\mathrm{R}$ & 8 \\
\hline \multicolumn{13}{|l|}{ Unicell: } \\
\hline Cr-TRP1 & $\mathbf{L}$ & I & G & $\mathbf{F}$ & $T$ & $\mathbf{F}$ & $\mathbf{N}$ & $\mathbf{F}$ & I & A & $\mathbf{F}$ & 6 \\
\hline Cr-TRP6 & $\mathbf{F}$ & $\mathbf{F}$ & G & $F$ & A & $\mathbf{Y}$ & $\mathbf{N}$ & $\mathbf{L}$ & v & A & $\mathbf{w}$ & 9 \\
\hline Cr-TRP13 & w & $\mathrm{E}$ & G & v & $\mathbf{F}$ & $Y$ & $\mathbf{N}$ & $\mathbf{L}$ & I & A & $\mathbf{Y}$ & 7 \\
\hline \multicolumn{13}{|l|}{ Non-TRPs: } \\
\hline TPCN1 I & $\mathbf{F}$ & $\mathbf{L}$ & G & $F$ & $Y$ & $\mathbf{F}$ & S & $\mathbf{L}$ & $\mathbf{L}$ & K & I & 6 \\
\hline TPCN1 II & $\mathbf{L}$ & $\mathbf{F}$ & $\mathrm{D}$ & $\mathbf{F}$ & $\mathbf{F}$ & $\mathbf{F}$ & I & V & A & $\mathbf{I}$ & I & 4 \\
\hline NavAb & $\mathbf{F}$ & $Y$ & - & $\mathbf{F}$ & $\mathbf{F}$ & $\mathbf{F}$ & $\mathbf{N}$ & V & V & A & $E$ & 7 \\
\hline Nav1.4 I & $\mathbf{Y}$ & $\mathbf{F}$ & $\mathrm{P}$ & $\mathbf{F}$ & $\mathbf{F}$ & $\mathbf{I}$ & $\mathbf{N}$ & I & $\mathbf{L}$ & A & K & 5 \\
\hline Nav1.4 II & $P$ & G & $P$ & $F$ & $\mathbf{F}$ & V & $\mathbf{N}$ & $\mathbf{F}$ & $\mathbf{L}$ & A & - & 4 \\
\hline Nav1.4 III & G & $\mathbf{F}$ & $E$ & $\mathbf{F}$ & $\mathbf{L}$ & $\mathbf{F}$ & $\mathbf{N}$ & $\mathbf{F}$ & I & G & M & 5 \\
\hline Nav1.4 IV & $P$ & $T$ & K & $Y$ & $\mathbf{F}$ & $\mathbf{Y}$ & $\mathbf{N}$ & $\mathbf{Y}$ & I & A & D & 6 \\
\hline KvAP & - & $\mathbf{Y}$ & S & $\mathbf{F}$ & $\mathbf{F}$ & A & $\mathbf{T}$ & $\mathbf{L}$ & $\mathbf{I}$ & G & - & 5 \\
\hline Kv1.2 & $Y$ & $F$ & $K$ & $\mathrm{~F}$ & $w$ & I & $\mathbf{Q}$ & V & A & S & $Q$ & 2 \\
\hline
\end{tabular}

Table 1. Conservation and identity of TRP fingerprint residues in different channels. Residue numbering corresponds to the rat TRPV1 sequence (poe TRPV1). TRP and nonTRP channels are presented. The number in the last column corresponds to the total number of fingerprint residues for each channel. For non-TRPs, sequence and structural alignments were used to determine equivalent positions. Green corresponds to identities while black represents homology. Red shades denote non-conserved residues. Minus signs represent gaps (non aligning residues). $\Phi$ denotes 6 carbon aromatic residues (i.e., Tyr or 154 Phe). 
157 TDh. The latter is considered an important modulator of TRP gating and although different

158 in sequence in TRPA1 channels when compared to other GI-TRPs, it is structurally

159 equivalent (Paulsen et al., 2015). Thus, our results suggest that such specific specialization

160 in TRPA originated after the divergence from the common ancestor shared with TRPVs and

161 is unlikely to be found outside this group. Within the unicellular group, crTRP1 shows only

1626 identities, that increases up to 7 and 10 for the case of the unicellulars that are closer to

163 TRPA and TRPV clades respectively (i.e., UC1 to 3 groups in Figure 1; Table 1).

164 There are some highly conserved residues that were not considered in our analysis

165 because they score just below the threshold. These include a highly conserved glycine

166 (88.1\%) commonly found at the selectivity filter, a phenylalanine $(85.1 \%)$ at the beginning

167 segment of TM5 and an aspartic acid (83,2\%) localized at the end of TM4-TM5 linker. The

168 latter two residues are in close proximity to L678 (according to rTRPV1 numbering) a

169 sidechain associated to channel response to both agonist and pH in different TRPs channels

170 (Boukalova et al., 2010; Du et al., 2009; Kasimova et al., 2017; Klausen et al., 2014).

171 Several groups have suggested an evolutionary relationship between TPCNs and TRP

172 channels (Clapham \& Garbers, 2005; Galione, 2010). TPCN channels are thought to be

173 asymmetric (Penny et al., 2016; Kintzer \& Stroud, 2017). In support of the argument of

174 asymmetry, while one domain (D1) shows 8 coincidences with the TRP fingerprint, the

175 other domain (D2) is more similar to eukaryotic voltage-gated sodium channels (Nav) with

176 only 5 coincidences. Interestingly, the monomeric bacterial channel NabAB (Payandeh et

177 al., 2011) shares seven of these eleven signature residues, and the mammalian Nav1.4 
178 exhibits only 6 residues in domain IV, which holds the highest number of hits compared to

179 domains I-III (Table 1). Thus, in our analysis, TPCN and Nav have the larger score of

180 similarities in residue conservation outside the TRP family. Overall, phylogenetic and

181 primary sequence analyses provide strong support for a fingerprint in TRP channels that is

182 composed of 8 to 11 non-contiguous residues (F $\mathbf{\Phi} \mathbf{G} \boldsymbol{\Phi} \boldsymbol{\Phi} \boldsymbol{\Phi} \mathbf{N} \mathbf{L}$ I A W).

\section{Sequence conservation highlights structural features.}

185 To visualize the position of these fingerprint residues, TRP channel structures from the

186 different families were compared (i.e., TRPV1, PDBID 5IRZ; TRPM8, PDBID 6BPQ; TRPC3,

187 PDBID 5ZBG; TRPA1, PDBID 3J9P and TRP1, PDBID 6PW5) (Gao et al., 2016; Yin et al.,

188 2017; Tang et al., 2018; Paulsen et al., 2015; McGoldrick et al., 2019). We identified three

189 well defined clusters (hereafter referred to as patches) of signature residues that are

190 present in all representative channels, including crTRP1 from green algae (Figure 3;

191 Supplementary Figure 2). Rat TRPV1 numbering was used throughout the manuscript to

192 identify amino acid positions.

193 The first patch (P1) gathers several residues from a hotspot that has been historically

194 linked to channel modulation. It is composed of side chains from TM1 (Phe434 [91.9\%]),

195 the TM4-TM5 linker (Gly563 [98.6\%]) and the TDh (Trp697 [92.4\%]) (Figure 3; Table 2).

196 Initially proposed as critical for TRPV1 channel activation (Gregorio-Teruel et al., 2014),

197 these glycine at the linker and tryptophan at the TDh have been reported as a common

198 theme in TRPs. In this context, the TDh seems to operate as an integrator, receiving 
bioRxiv preprint doi: https://doi.org/10.1101/2 21.09.06.459056; this version posted Septen per 6, 2021. The copyright holder for this preprint (which was not certified by peer review) is th author/funder who has graftedbioRxin alice, se to display the preprint in perpetuity. It is made

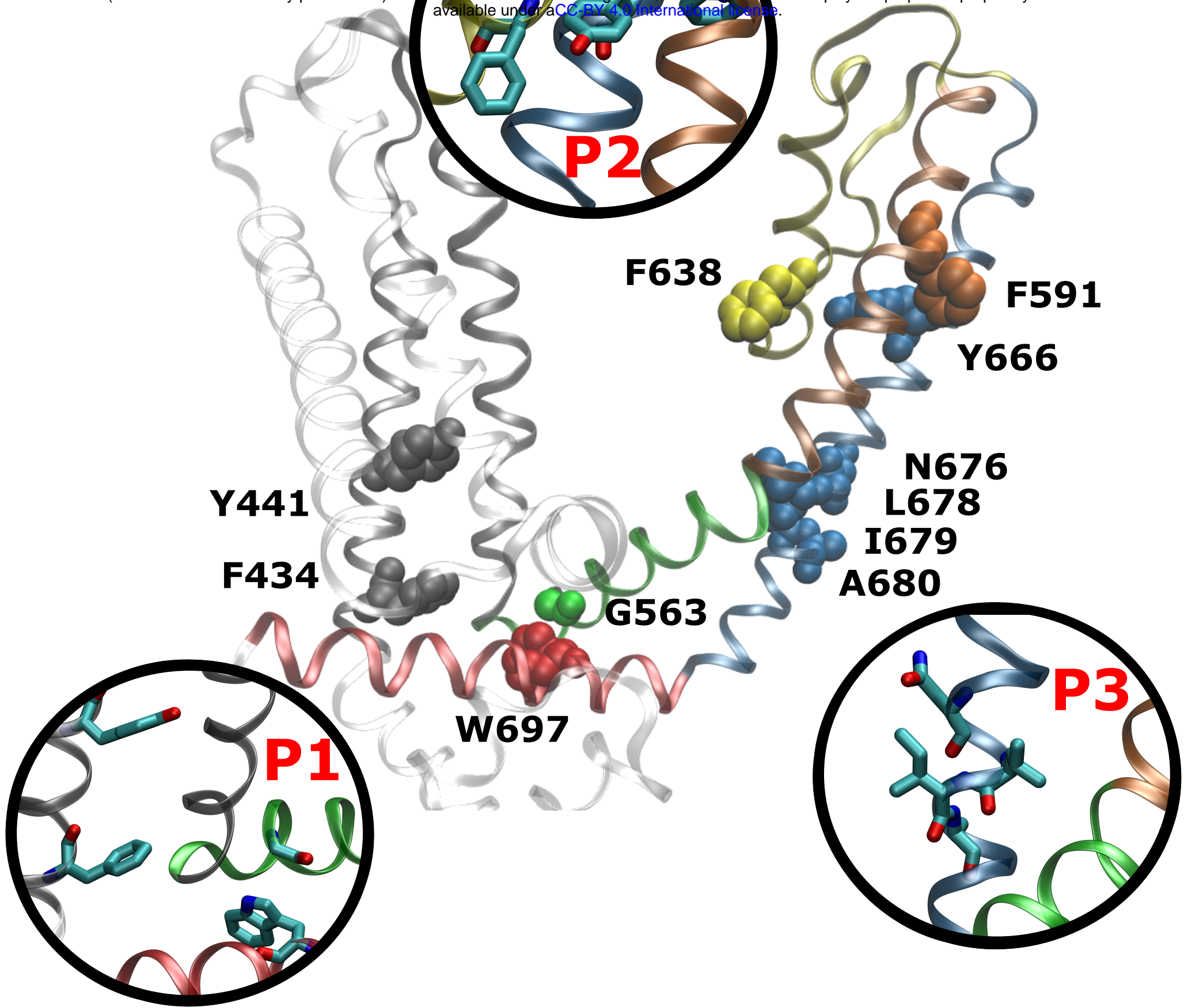

Figure 3. Spatial distribution of TRP channel fingerprint. Highly conserved residues are arranged in three well-defined patches, highlighted as insets and dubbed P1, P2, and P3. The structural data and residue numbering corresponds to rat TRPV1 (PDB:5IRZ). For clarity only one protomer is shown. Backbone and residues follow the code color used in Figure 2. 
199 information from lipids (such as PIP2), the TM4-TM5 linker that reports changes occurring

200 at the transmembrane region and the coupling domain (CD) composed of the pre-S1 helix

201 and a helix-loop- helix (HLH) motif. The high conservation of Phe434 is underscored by the

202 importance of the CD array that couples with all the rest of the cytoplasmic

203 features (Garcia-Elias et al., 2015; Romero-Romero et al., 2017; Hofmann et al., 2017; Yang

204 et al., 2018; Hilton et al., 2019; Yuan, 2019; Zubcevic et al., 2019; Cao, 2020).

205 The second patch (P2) is located at the selectivity filter region and is composed of three

206 phenyl group residues, i.e., Phe591 [Ф 97.4\%], Phe638 [Ф 97.2\%], and Tyr666 [Ф 98.7\%],

207 located at both the TM6 helix and the pore helix (Figure 3). A glycine residue that forms

208 part of the selectivity filter/upper constriction in TRPs is also highly conserved (87.9\%)

209 but slightly off to the $90 \%$ threshold (Figure 2). The high conservation of these residues

210 within the selectivity filter contrasts with the high variability observed by us and others at

211 the pore region (Huffer et al., 2020).

212 A third patch (P3) is localized at the lower portion of the pore and is composed of a well-

213 studied set of residues forming the lower gate (Asn676 [95.0\%], Ile679 [90.4\%], Ala680

$214[94.4 \%])$ as well as Leu678 [95.4\%] that is facing the interface between TM5 and TM6

215 (Palovcak et al., 2015) (Figure 3).

216 Finally, we identified a prevalent aromatic side chain (Tyr441 [76.6\% Tyr $+15.2 \%$ Phe]),

217 located at the middle of the TM1 helix. This residue was not observed in close contact to

218 any other signature residue (Figure 3). 
219 From the structural data, the most conserved interaction between signature residues is

220 between the glycine (Gly563) in the TM4-TM5 linker and the tryptophan (Trp697) at the

221 TDh. This observation is further supported by our evolutionary coupling analysis showing

222 that the highest score for putative interactions -within the region we studied- are precisely

223 those established between the TM4-TM5 linker and the TDh (Supplementary Figure 3). The

224 analysis also showed interactions linking the lower portion of TM2 to the end of the TDh.

225 Such interaction cannot be explained by direct contact between TM2 and TDh. Thus, it

226 would not be unreasonable to suggest that this interaction between TM2 and the TDh

227 involves an additional linker molecule such as $\mathrm{PIP}_{2}$ or other lipid binding to this

228 region (Poblete et al., 2014; Yin et al., 2017; Yazici et al., 2021; Hughes et al., 2018) ).

229 Interactions between the channel and the membrane at the cytosol-membrane interface is

230 emerging as a common theme in TRPs. Under this view, different parts of the CD/TDh

231 coupling mechanism are tuned by the differences in binding of membrane lipids and/or

232 canonical ligands (reviewed in Zubcevic, 2020). Moreover, a calcium binding site located at

233 the intracellular linker has been observed in several TRP channel structures and associated

234 with channel function (Brauchi \& Rothberg, 2020). To our knowledge, calcium-dependent

235 modulation of the putative TM2-lipid-TDh interaction has not been explored in detail.

236 Series of independent studies had reported that mutations of residues that form part of the

237 fingerprint -or the connecting side chains- modify or impairs channel activity, underscoring

238 their importance in maintaining proper channel activity (Table 2). Then, we analyzed the

239 networks of connectivity between the signature residues in channels from different 


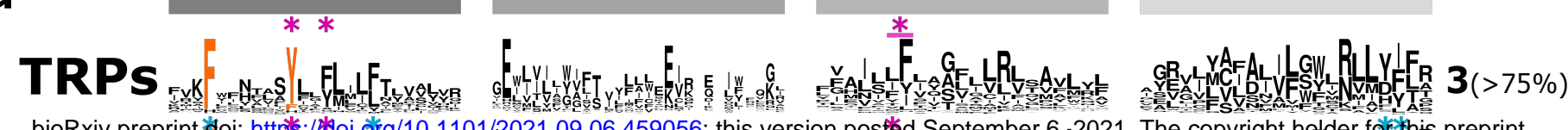

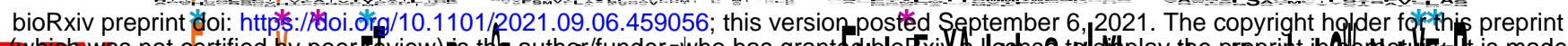

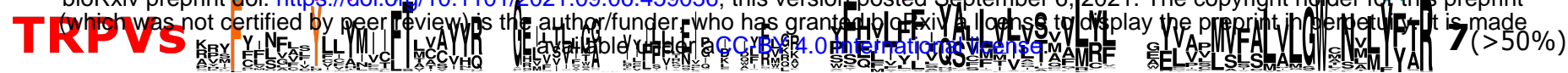

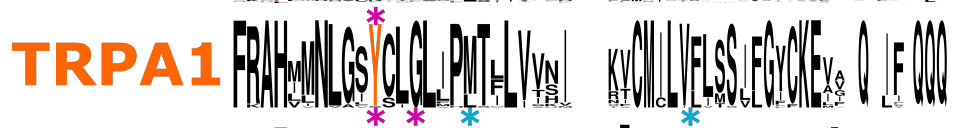

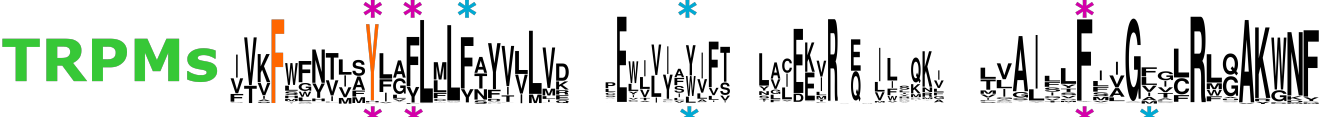

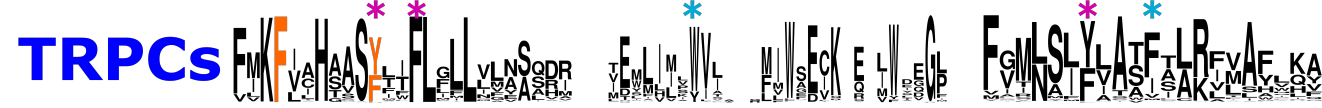

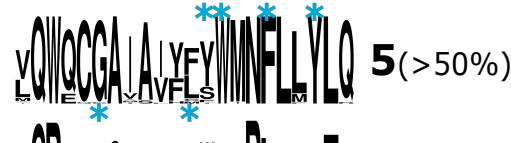
(U)

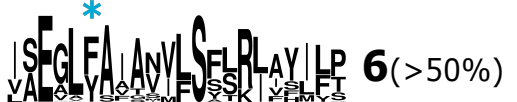

b

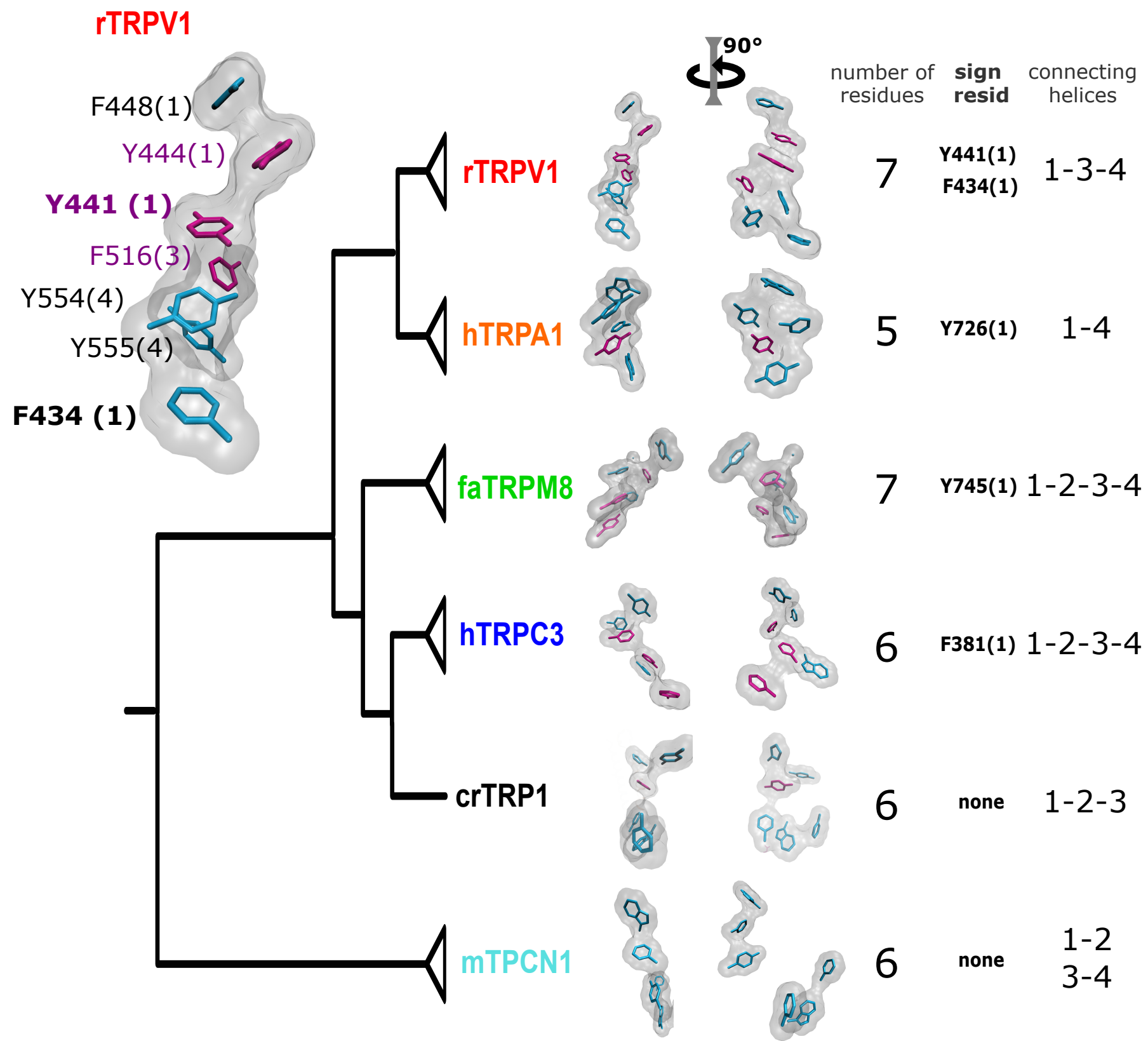

Figure 4. Aromatic residue distribution in LBD. All the highlighted positions comply two conditions, i.e., to be facing the core of the LBD, and to be at $<4.5 \AA$ from another aromatic residue. a) Conservation of aromatic residues on transmembrane helices 1 to 4 . Orange letters: the two fingerprint aromatics in TM1. Violet asterisk: $>50 \%$ of aromatic conservation within subfamilies. Cyan asterisks: $>50 \%$ of aromatic conservation within the subfamily. b) Comparison between AC volumes presented next to a schematic view of the topology obtained in our phylogenetic analysis. Cyan licorice: conserved residues $(>50 \%)$ in specified subfamily, Magenta licorice: conserved residue $(>50 \%)$ in all families. Inset. Aromatic core in rTRPV1. The specific positions of the aromatics are indicated. Structures used: rTRPV1, PDB: 5IRZ; faTRPM8, PDB: 6BPQ; hTRPC1, PDB: 5ZBG; hTRPA1, PDB: 3I9P; crTRP1, PDB:6PW5; mTPCN1, PDB: 6C96 
240 subtypes. The apo and ligand-bound state conformations of prototypical channels were

241 used to compare state-dependent changes in connectivity (i.e., TRPV1/2/3/5/6,

242 TRPC3/4/6, TRPM2/4/8 and TRPA1). As reported abundantly in the literature, the

243 signature residues in the TM4-TM5 linker and TDh from P1 and the residues in P3 forming

244 the lower gate experience changes in their network of connectivity (Supplementary Figure

245 4). On the other hand, residues in P2 appear displaced altogether as result of pore

246 reconfiguration with just minor changes in distances between them (Supplementary Figure

$2474)$.

249 A conserved aromatic core at the transmembrane region of TRPs.

250 Four phenyl groups (i.e., Phe/Tyr) were identified in specific positions within the

251 transmembrane domain in more than $75 \%$ of the sequences. Further inspection of

252 structural data showed that these residues are part of a larger cluster of aromatic amino

253 acids, common to all TRP channel structures (Figure 4). A group of six to seven aromatic

254 residues belonging to the VSLD/LBD domain (including at least one signature residue from

255 P1), appear to form an aromatic core (AC) (Figure 4 a and b). In contrast, voltage-gated

256 potassium and sodium channels occupy these positions with charged amino acids forming

257 salt bridges, short aliphatic side chains, or aromatics located at the membrane-water

258 interface (Supplementary Figure 5). Although the overall three-dimensional shape of the

259 AC varies, it is present in all TRP subtypes, connecting three to four helices from within 
260 the VSLD/LBD, suggesting they serve as scaffold that is holding together the whole

261 VSLD/LBD domain (Figure 4b).

262 The AC emerges as a common theme present from crTRP1 to TRPV1-4. The most dramatic

263 case is observed in the TRPV1-4 clade, exhibiting both the highest number of aromatics (7)

264 and the most ordered stack (Figure 4 b). In contrast, TRPA1 shows only 5 aromatics

265 forming the AC and a wider disposition, with only two helices connected through the

266 aromatic network. Although the residues in TRPA1 are still in close contact, they do not

267 form the compact stacking observed in other TRPs, including crTRP1.

268 The presence of these conserved aromatics next to ligand binding sites -or even forming

269 part of it- suggests a mechanism in which the AC acts by imparting rigidity to the region

270 and functionally linking the transmembrane helices 1-4, facilitating the translation of

271 mechanical force from the ligand binding sites to the coupling domains that connect

272 multiple parts of the channel including the TDh, cytoplasmic CD, and preTM1. Minimal or

273 no displacement among these aromatics was observed by comparing the apo and ligand-

274 bound structures from different TRP members (Supplementary Figure 4). This suggests to

275 us that their role might be related to imposing rigidity to the VSLD. Rigidity that might be

276 affected by ligand binding as part of a process that has been tuned during evolution in the

277 different subfamilies. Moreover, consistent with the notion that TPCN channels are close

278 relatives of the TRP family, the presence of a group of 6 aromatics facing inside the VSLD is

279 conserved in TPCN's domain 1 and absent in domain 2 that is devoid of aromatics, 
280 resembling Nav channels. Unlike TRPs, this AC in TPCN channels looks less cohesive, or

281 “disconnected" (Figure 4b).

283 Conserved residues at the interaction between subunits.

284 TRP channels show a domain-swapped configuration. That is, the VSLD/LBD of one subunit

285 appears in close contact with its pore domain (PD) through the protein backbone and, at

286 the same time, in close contact with the PD of the neighboring subunit. The coupling

287 between the VSLD and the pore domain from different subunits of the tetramer is one topic

288 that has been poorly studied in TRP channels and not well understood in VGICs (Carvalho-

289 de-Souza \& Bezanilla, 2019; Shem-Ad et al., 2013; Neale et al., 2003).

290 By inspecting the structural data, we found a conserved interaction formed by a residue at

291 the middle of TM4 and a residue that is consistently preceding a signature residue in TM5

292 (Phe591 in rTRPV1; Figure 5a and b). Although the nature of the interaction varies, it is

293 present in all surveyed structures. Such interaction would put in direct contact TM helices

2944 and 5 from different subunits (i.e. the VSLD and the pore). By extension, this interaction

295 would communicate patches P1 and P2 from different subunits through the aromatics

296 running alongside the VSLD/LBD. The high conservation of these residues and their

297 relative positions within the structure suggests a common mechanism in TRPs where the

298 selectivity filter (P2) would be functionally connected to the ligand binding site located on

299 a neighboring transmembrane region. 

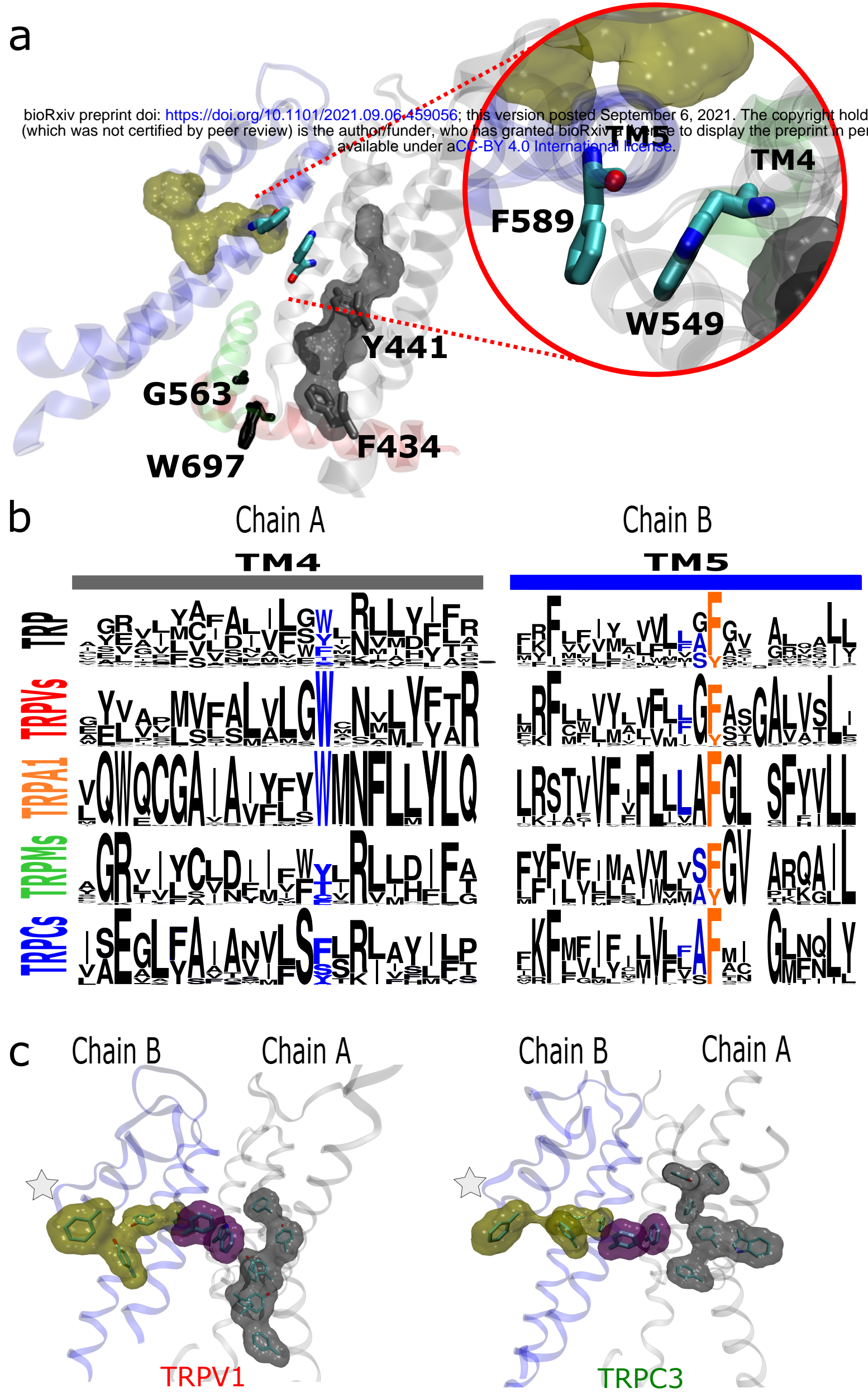

Figure 5. The AC connects to aromatics in P2 from neighboring subunits. a) A conserved intermolecular connection between residues (licorice) in helices at opposite faces to the AC (gray surface) and P2 (yellow surface). Signature residues from P1 are depicted in gray (F434 and Y441) and black (G563, and W697). Inset: Upper view of residues establishing the inter-subunit interaction (PDB:5IRZ). b) Residues connecting TM4 and TM5 from neighboring subunits align on the same position in TM4 and at one or two positions from the 4th fingerprint residue in TM5. c) Lateral view of the interaction between LBD and PD from different subunits in the most distant members of the TRP clade. 


\section{DISCUSSION}

301 In the present work we studied the conservation and frequency distribution of amino acids

302 within the transmembrane of TRP channels and flanking regions, trying to conciliate

303 structural and evolutionary contexts (Figure 6). A highly conserved set of residues are

304 located and grouped at strategic positions within the channel's transmembrane region,

305 defining a fingerprint for GI-TRPs. Moreover, a conserved network of interactions connects

306 them directly or indirectly.

307 From the three patches described in this article, the aromatics forming patch 2 were not

308 described in the literature as such. Nevertheless, previous studies showed the importance

309 of the residues composing this patch. Phe591 is described as forming part of the higher,

310 wider region of the capsaicin pocket binding in TRPV1 channels (Elokely et al., 2015) and

311 mutations at Tyr638 and Tyr666 have obvious effects on channel activity. Specifically,

312 Tyr666Ala is not functional in TRPV1 (Susankova et al., 2007), the equivalent mutant

313 Tyr661Cys in TRPV3 is not activated by temperature, but still responds to agonist (Grandl

314 et al., 2008), and the Tyr702Leu equivalent mutant in TRPV4 has significantly reduced

315 responses to agonists, temperature, and mechanical stimulation (Klausen et al., 2014).

316 Moreover, mutations at equivalent positions to rTRPV1 Tyr638 in channels from different

317 subfamilies elicit a wide range of effects from gain-of-function to dominant-negative

318 phenotypes (Munns et al., 2015; Kitaguchi \& Swartz, 2005; Bidaux et al., 2015; Strübing et

319 al., 2003; Chandrabalan et al., 2019; Vinayagam et al., 2018; Paulsen et al., 2015).

320 Noteworthy, Bidaux et al., (Bidaux et al., 2015) demonstrated that mutations at this 


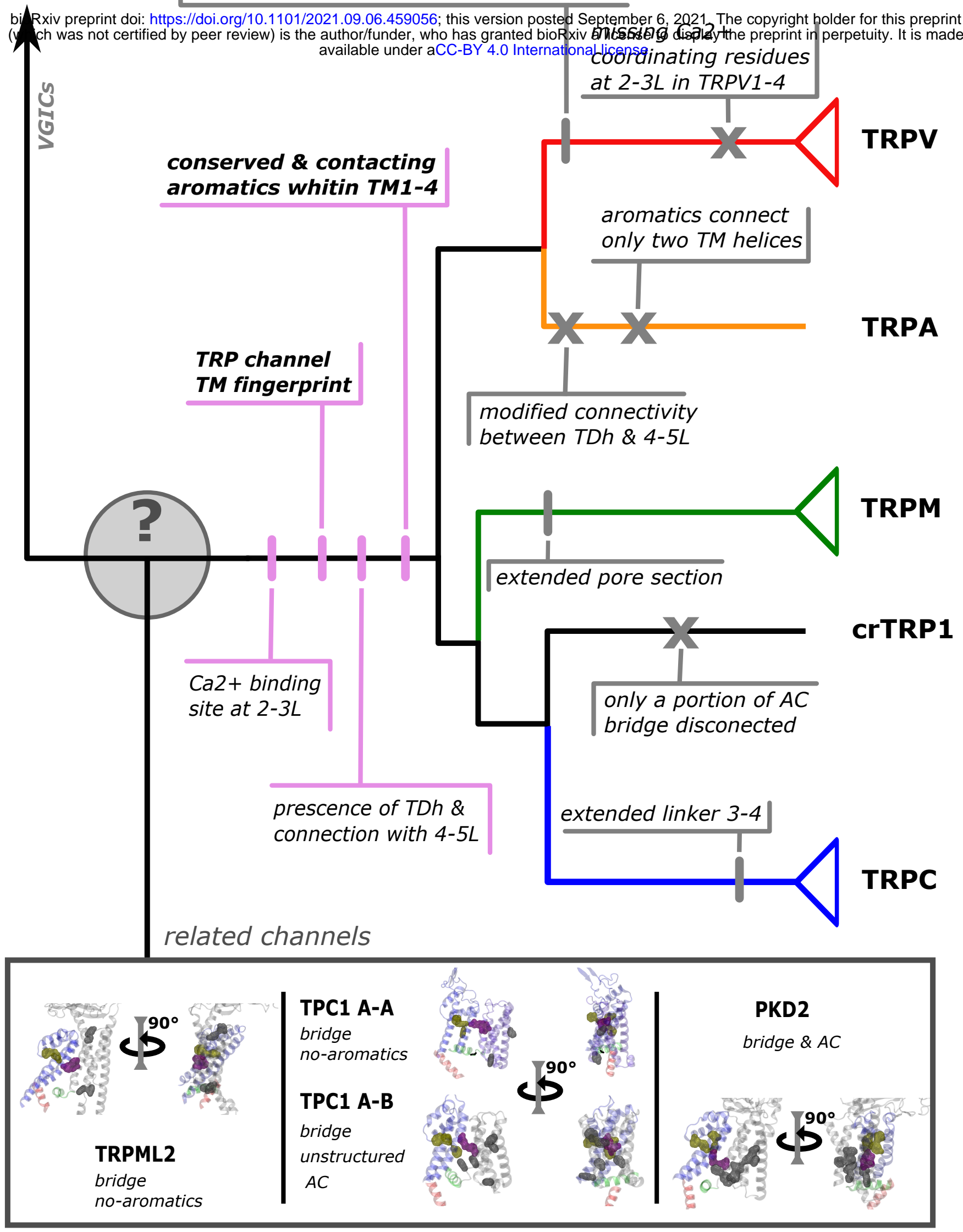

Figure 6. Conserved modif ications observed in TRP proteins. The different channels studied in this work are presented next to a schematic view of the topology obtained in our phylogenetic analysis. Unique TRP features are highlighted in pink shades. Other features that appeared segregated by families are highlighted in grey shades. 
321 position (Tyr908 in TRPM8) to Ala or Trp abolish the response to temperature and

322 menthol but not to icilin, yet mutation to Phe keeps the channel fully functional. As shown

323 here, the three aromatics forming P2 always need a non-conserved fourth hydrophobic

324 residue to connect them all. By comparing apo and ligand bound structures, P2 consistently

325 seems to translate as if it were a near-rigid-body, moving as a whole compact structure.

326 Our analysis suggests that the function of this patch might be associated with the

327 communication between the VSLD/LBD and the selectivity filter from different subunits.

328 Residues on the other two patches have been recurrently studied in the literature. In

329 particular, residues of patch 3 have been characterized as shaping the lower gate of TRP

330 channels and to participate in stabilizing the transition between $\alpha$ - and $\pi$-helix types during

331 opening (Palovcak et al., 2015; Kasimova et al., 2017; Kasimova et al., 2018).

\section{The interaction between signature residues and the aromatic core}

334 Two of the residues in the P1 patch have been proposed as fundamental to the interaction

335 between the TDh and the TM4-TM5 linker, acting as an allosteric integrator (Taberner et

336 al., 2014; Sierra-Valdez et al., 2018; Romero-Romero et al., 2017; Gregorio-Teruel et al.,

337 2014; Zhao et al., 2020). A third residue in P1 corresponds to a conserved Phe at the lower

338 end of TM1 that is always connected to the other elements of the patch. Separated by 2

339 intermediate interactions, TRPM8 displays the largest distance between these two

340 components of patch P1. Notably, the gap observed between these two components has 
341 been associated to the binding site of menthol (Bandell et al., 2006; Malkia et al., 2009).

342 Moreover, the menthol analog WS-12 binds to Tyr745 (TM1), and sits close to Tyr1004

343 (TDh), seemingly reinforcing the connection between the VSLD/LBD and the end of the

344 TDh in TRPM8 (Yin et al., 2019).

345 There is only one signature residue that is consistently forming part of the aromatic core

346 (i.e., Tyr441 in TRPV1). Mutations to Ser of Tyr441 in TRPV1 generates non-functional

347 channels (Boukalova et al., 2013). The equivalent residue in TRPM3 channels presents

348 impairments in response to agonists when mutated from Tyr to Thr, and a Tyr to His

349 mutation in TRPM8 channels has been shown critical to both menthol activation and the

350 inhibitory effect of the small molecule SKF96365 (Bandell et al., 2006; Malkia et al., 2009).

351 This coincided with similar phenotypes observed by mutating other residues belonging to

352 the AC. This is the case of Tyr444 that in TRPV1 generates non-functional

353 channels (Boukalova et al., 2013) and the double mutation Y885T / W982R in TRPM8 that

354 presented altered responses to agonists and temperature (Held et al., 2018).

355 When comparing the disposition of the aromatic core in detail, it appears obvious they

356 have different distributions in the different TRP channel families. However, certain

357 generalizations can be drawn. The AC appears associated with P2 at the selectivity filter

358 and disconnected from P1 in most families. Notably, in TRPVs the AC extends down to

359 reach the lower aromatic residues of P1 making a direct connection between the AC and

360 the TDh / TM4-TM5 linker. Hence, a P2-AC-P1 continuum can be observed in the TRPV

361 subgroup. Interestingly, from the structures obtained in the presence of agonists it 
362 becomes evident that these agonists bridge the AC and P1 (e.g., WS-12 in TRPM8 and GFB-

3639289 in TRPC4; PDBIDs 6NR2 and 7B16, respectively; Yin et al., 2019; Vinayagam et al.,

364 2020). The disposition of the components of $\mathrm{P} 1$ (i.e., TM1;TM4-TM5linker;TDh) and

365 differences in the AC suggest similar but nonidentical coupling strategies among GI-TRPs.

366 The presence of an aromatic core, connecting the transmembrane helices of the ligand

367 binding pocket and communicating critical modulatory regions that are far apart in the

368 structure (e.g., the selectivity filter and the TDh) appears as a modular connector that has

369 been subject to variations throughout evolution. This suggests a common aspect of TRP

370 channel mechanics that remains largely unexplored. Our line of reasoning also suggests

371 that the observed rearrangements of the selectivity filter during activation are likely linked

372 to inter-subunit interactions, likely modulated by the AC. At the same time, it implies that

373 certain ligands might have the ability to modulate the conformation of the selectivity filter -

374 and by extension the extracellular linkers- without the need of an open gate conformation.

376 METHODS

377 Amino Acid sequences, alignment, and phylogenetic analyses. Protein sequences

378 corresponding to TRPA1, TRPC1, TRPC3-7, TRPM1-8, TRPML1-3, TRPP1-3, and TRPV1-6

379 from human (Homo sapiens), TRPC2 from the house mouse (Mus musculus), NompC from

380 the fruit fly (Drosophila melanogaster) and TRPY1 from the brewing yeast (Saccharomyces

381 cerevisiae) were retrieved from the International Union of Basic and Clinical Pharmacology 
382 (IUPHAR) database. The transmembrane regions of these sequences were used as queries

383 in blastp searches (Altschul et al., 1990) against the proteomes of Chlamydomonas

384 reinhardtii, Volvox carteri, Coccomyxa subellipsoidea, Micromonas pusilla, Dictyostelium

385 discoideum, Dictyostelium purpureum, Leishmania infantum, Leishmaniamajor, Leishmania

386 mexicana, Paramecium tetraurelia, and Trypnosoma cruzi to search for putative TRP

387 channels. Putative channels were selected based on the frequency of hits to the query

388 sequences relative to human, friut fly, and yeast. This was followed by reciprocal blastp

389 searches (E-value $<1 \mathrm{e})$, and a final inspection for the presence of the TRP domain. To

390 investigate the phylogenetic position of these candidate TRP channels we retrieved protein

391 sequences corresponding to TRPVs, TRPA1, TRPMs, TRPN1, and TRPCs in representative

392 species of all main lineages of amniotes (Supplementary Table S1) from the Orthologous

393 MAtrix project (OMA) (Altenhoff et al., 2021). Thus our final dataset included 844 protein

394 sequences from bona fide TRP channels from amniotes in addition to 24 sequences from

395 unicellular organisms. Amino acid sequences were aligned using MAFFT v.7 (Katoh et al.,

396 2017) allowing the program to choose the alignment strategy (FFTNS2). We used the

397 proposed model tool of IQ-Tree v.1.6.12 (Minh et al., 2020) to select the best-fitting model

398 of amino acid substitution (JTT+G4). We used the maximum likelihood method to obtain

399 the best tree using the program IQ-Tree v1.6.12 (Minh et al., 2020). We carried out 3

400 independent runs to explore the tree space, and the tree with the highest likelihood score

401 was chosen. We assessed support for the nodes using the ultrafast bootstrap routine as

402 implemented in IQ-Tree v1.6.12 (PMID: 29077904). Human (Homo sapiens) and chicken

403 (Gallus gallus) Potassium voltage-gated channel subfamily A member 2 (KCNA2), Sodium 
404 Voltage-Gated Channel Alpha Subunit 8 (SCN8A) amino acid sequences were included as an 405 outgroup.

406 MSA database. 474 extra sequences from the Uniprot protein database were added to the 407 pool of sequences rescued from the OMA server and then realigned in MAFFT. The region 408 corresponding to the transmembrane was identified, and the rest of the sequence was 409 removed, leaving the section from the last portion of the pre-TM1 region to the last residue 410 of the TRP helix (using rTRPV1 as reference). A second round of sequence alignment in 411 MAFTT (FFTNS1 strategy) was performed and manually refined to minimize gaps. The 412 resulting database contains 1311 sequences, 837 selected from OMA database and 413 identified unicellulars and 474 from Uniprot, including sequences annotated as TRP or 414 TRP-like channels and several uncharacterized protein sequences.

415 Coevolution analysis. Coevolution scores were calculated using asymmetric pseudo416 likelihood maximization direct coupling analysis algorithm (aplmDCA), (Ekeberg et al., 417 2013). This algorithm finds the approximate parameters of the maximum entropy 418 probabilistic model consistent with selected MSA statistics (univariate and bivariate 419 frequency distributions). Default parameters were used for field and coupling 420 regularization and sequence reweighting (lamba_h $=0.01$, lambda_j $=0.01$, theta $=0.1$ ).

421 Structures. The structures used were rTRPV1, PDB entry 5IRZ; faTRPM8, 6BPQ; hTRPC3, 422 5ZBG; hTRPA1, 3J9P; crTRP1, 6PW5; mTPCN1, 6C96; rKv1.2, 3LUT and hNav1.4, 6AGF. To 423 compare the apo vs ligand-bound/activated channel structures, the PDB files used were 
424 PDB:5IRZ -apo-, PDB:5IRX -RTX +DkTx-), Ficedula albicollis TRPM8 (PDB:6BPQ -apo-; PDB:

425 6NR2 -Ws-12 + PIP2-) and Danio rerio TRPC3 (PDB: 6G1K -apo-; PDB:7B16 -GFB-9289-).

426 Figure preparation. To visualize the identities and gap patterns on the MSA, images were

427 exported using Jalview 2.11.1.3 (Waterhouse et al., 2009). The sequence logos show the

428 distribution of amino acid residues at each position in the regions of interest, and were

429 generated using WebLogo version 3 (Crooks, 2004). The structural figures were generated

430 using VMD 1.9.2 (Humphrey et al., 1996). Direct interactions between residues were

431 identified using a distance threshold of $4 \AA$, except for the pi-pi interactions, where a $5 \AA$

432 threshold was used (Piovesan et al., 2016).

436 Keywords: MSA, evolution, allosterism, structure.

437 Acknowledgements: We acknowledge Dr. Gonzalo Riadi from Universidad de Talca for 438 providing computer support. JCO acknowledges the Integrative Biology Group members, 439 Universidad Austral de Chile, for their constant support, scientific enthusiasm, and creative 440 feedback. This work was supported by Fondo Nacional de Desarrollo Científico y 441 Tecnológico from Chile (FONDECYT 1191868) to SB, (FONDECYT 1210471) to JCO, and 442 ANID-Millennium Science Initiative Program \#NC160011 (SB and JCO).

444 Author contribution: SEB, CKC, and DCB designed experiments and analyzed data. VC, KZ 445 and JCO designed the taxonomic sampling, retrieved amino acid sequences and performed 446 phylogenetic analyses. DCB and SEB prepared the figures. DCB, CKC, SEB and JCO wrote the 447 manuscript. 
Table 2. Summary of structural-functional studies and reported effects of site directed mutagenesis in signature residues. First column indicates the equivalent signature residue in the rTRPV1 sequence. Second column indicates the channel member studied. Third column describes the amino acid identity or mutation, and fourth column corresponds to the type of study used to determine functional effects. MDS: Molecular dynamics simulations, SDM: Sitedirected mutagenesis. Effects and references are described in the last two columns.

\begin{tabular}{|c|c|c|c|c|c|}
\hline $\begin{array}{l}\text { TRPV1 } \\
\text { Position }\end{array}$ & Channel & Residue & $\begin{array}{l}\text { Evidence } \\
\text { source }\end{array}$ & Effect & Refference \\
\hline \multirow[t]{2}{*}{ F434 } & drTRPC4 & F366 & Structure & Part of cholesterol binding pocket. & Vinayagam et al.,2018 \\
\hline & hTRPV1 & H719A & SDM & Disrupt the voltage-gated opening & Zimova et al.,2018 \\
\hline \multirow[t]{3}{*}{$F / Y 441$} & TRPV1 & Y441S & SDM & Non functional & Boukalova et al.,2013 \\
\hline & TRPM8 & Y745H & SDM & Critical on Menthol Sensitivity. & Bandell et al.,2006 \\
\hline & & Y745H & SDM & $\begin{array}{l}\text { Critical on inhibition SKF96365-mediated of Cold- } \\
\text { and voltage- activation, but just partially on other } \\
\text { inhibitor }\end{array}$ & Malkia et al.,2009 \\
\hline \multirow[t]{8}{*}{ G563 } & rTRPV1 & G563S/C & SDM & Gain of Function & Boukalova et al.,2010 \\
\hline & & G563S/A & SDM & $\begin{array}{l}\text { Gain of Function, Inhibition by proton of Max } \\
\text { current induced by capsaicin }\end{array}$ & Boukalova et al.,2013 \\
\hline & mTRPV1 & G564S & SDM & Gain of Function & Duo et al.,2018 \\
\hline & rTRPV3 & G573S/C & SDM & Gain of Function & Xiao et al.,2008 \\
\hline & & G573S/C & SDM & Gain of Function, Olmsted Syndrome & Lin et al.,2012 \\
\hline & $\mathrm{mTRPC} 4 / 5$ & $\begin{array}{l}\text { G503S/ } \\
\text { G504S }\end{array}$ & SDM & Gain of Function & Beck et al.,2013 \\
\hline & TRPC3 & G552 & Structure & Coupled W673 from TRP domain & Fan et al.,2018 \\
\hline & TRPCh & G552 & Structure & Coupled W673 from TRP domain & Fan et al.,2018a \\
\hline \multirow[t]{3}{*}{ F/Y591 } & rTRPV1 & F591 & MDS & Part of the vanilloid binding pocket & Elokely et al.,2015 \\
\hline & & F591A & SDM & $\begin{array}{l}\text { Low Capsaicin response, non response to } \mathrm{pH} \text { and } \\
\text { not } \mathrm{RTX} \text { binding }\end{array}$ & Ohbuchi et al., 2016 \\
\hline & hTRPM4 & Y944 & Structure & Forming face to face $\pi$-stack with F1027 on TM5 & Duan et al.,2018 \\
\hline \multirow[t]{6}{*}{$F / Y 638$} & rTRPV1 & F638A & SDM & Gain of Function, NMDG/Na selectivity rise & Munns et al.,2015 \\
\hline & & F638W & SDM & $\begin{array}{l}\text { Enhanced the sensitivity to the acylpolyamine } \\
\text { toxins AG489 and AG505 }\end{array}$ & Kitaguchi et al.,2005 \\
\hline & TRPM8 & Y908A/W & SDM & $\begin{array}{l}\text { Not responsive to Cold and Menthol but } \\
\text { responsive to Icilin }\end{array}$ & Bidaux et al., 2015 \\
\hline & & Y908F & SDM & Totally responsive to Cold and Menthol and Icilin & Bidaux et al.,2015 \\
\hline & TRPC4 & F572 & Structure & $\begin{array}{l}\text { Stabilizes the pore through an hydrophobic } \\
\text { contact with neighbour protomer }\end{array}$ & Vinayagam et al.,2018 \\
\hline & mTRPC5 & F576A & SDM & Non-functional, dominant negative & Strübing et al.,2003 \\
\hline
\end{tabular}




\begin{tabular}{|c|c|c|c|c|c|}
\hline & hTRPA1 & F909A & SDM & $\begin{array}{l}\text { Affect different agonists and antagonists } \\
\text { responses }\end{array}$ & $\begin{array}{l}\text { Chandrabalan et } \\
\text { al.,2019 }\end{array}$ \\
\hline & & F909T & SDM & $\begin{array}{l}\text { Abolish the A-967079-inhibition of AITC-evoked } \\
\text { response }\end{array}$ & Paulsen et al.,2015 \\
\hline \multirow[t]{6}{*}{ Y/F666 } & TRPV1 & Y666A & SDM & Non-functional (present in membrane) & Susankova et al.,2007 \\
\hline & mTRPV3 & Y661C & SDM & $\begin{array}{l}\text { Not responsive to Temp, but responsive to } \\
\text { agonist (2-APB and Camphor) }\end{array}$ & Grandl et al.,2008 \\
\hline & hTRPV4 & Y702L & SDM & Not responsive to Temp, Agonist and Swelling & Klausen et al.,2014 \\
\hline & hTRPM6 & Y1053C & SDM & $\begin{array}{l}\text { Causes hypomagnesemia with secondary } \\
\text { hypocalcemia, Decreased Current amplitud in } \\
\text { heterologus expresion in HEK293 }\end{array}$ & Lainez et al.,2013 \\
\hline & hTRPM4 & F1027 & Structure & Forming face to face $\pi$-stack with Y944 on TM5 & Duan et al.,2018 \\
\hline & hTRPA1 & F909A & SDM & Affect different agonist responses & $\begin{array}{l}\text { Chandrabalan et } \\
\text { al.,2019 }\end{array}$ \\
\hline \multirow[t]{3}{*}{ N676 } & rTRPV1 & N676 & MDS & Gating relies on the rotatory motion of $\mathrm{N} 676$ & Kasimova et al.,2018 \\
\hline & & N676A & SDM & Non-functional (present in membrane) & Kasimova et al.,2018 \\
\hline & & N676F & SDM & $\begin{array}{l}\text { Not responsive to Temp and Agonist (Cap/RTX) } \\
\text { and reduced response to } \mathrm{pH}\end{array}$ & $\begin{array}{l}\text { Kuzhikandathil et } \\
\text { al.,2001 }\end{array}$ \\
\hline \multirow[t]{4}{*}{ L678 } & rTRPV1 & L678A & SDM & $\begin{array}{l}\text { Low response to Agonist (Cap) and Temp, but } \\
\text { normal response to co-treatment }\end{array}$ & Susankova et al.,2007 \\
\hline & & L678P & SDM & $\begin{array}{l}\text { Not responsive to Temp and Agonist (Cap/RTX) } \\
\text { and reduced response to } \mathrm{pH}\end{array}$ & $\begin{array}{l}\text { Kuzhikandathil et } \\
\text { al.,2001 }\end{array}$ \\
\hline & TRPV3 & L768F & SDM & $\begin{array}{l}\text { Olmsted Syndrome and Erythromelalgia (gain of } \\
\text { function) }\end{array}$ & Duchatelet et al.,2014 \\
\hline & TRPC3 & L654 & Structure & Constriction site in the lower region of the pore. & Fan et al.,2018a \\
\hline \multirow[t]{11}{*}{1679} & rTRPV1 & 1697 & Structure & Constriction site in the lower region of the pore. & Liao et al.,2013 \\
\hline & & 1697 & Structure & Constriction site in the lower region of the pore. & Cao et al.,2013 \\
\hline & & 1697 & Structure & Constriction site in the lower region of the pore. & Gao et al.,2016 \\
\hline & & 1697 & Structure & Constriction site in the lower region of the pore. & Chugunov et al.,2016 \\
\hline & & 1697 & Structure & Constriction site in the lower region of the pore. & Susankova et al.,2007 \\
\hline & mTRPM4 & 11036 & Structure & Constriction site in the lower region of the pore. & Guo et al.,2017 \\
\hline & hTRPM4 & I1040 & Structure & Constriction site in the lower region of the pore. & Autzen et al.,2017 \\
\hline & drTRPC4 & 1617 & Structure & Constriction site in the lower region of the pore. & Vinayagam et al.,2018 \\
\hline & TRPV4 & $1715 N$ & SDM & $\begin{array}{l}\text { Hydrophobic single-residue gate. Higher resting } \\
\text { currents. }\end{array}$ & Zheng et al.,2018 \\
\hline & TRPC4 & I617N & SDM & $\begin{array}{l}\text { Hydrophobic single-residue gate. Higher resting } \\
\text { currents. }\end{array}$ & Zheng et al.,2018 \\
\hline & TRPM8 & V976S & SDM & $\begin{array}{l}\text { Hydrophobic single-residue gate. Higher resting } \\
\text { currents. }\end{array}$ & Zheng et al.,2018 \\
\hline A680 & TRPV1 & A680 & MDS & Change of Solvatation & Chugunov et al.,2016 \\
\hline
\end{tabular}


bioRxiv preprint doi: https://doi.org/10.1101/2021.09.06.459056; this version posted September 6, 2021. The copyright holder for this preprint (which was not certified by peer review) is the author/funder, who has granted bioRxiv a license to display the preprint in perpetuity. It is made available under aCC-BY 4.0 International license.

\begin{tabular}{|c|c|c|c|c|c|}
\hline & TRPV4 & A716S & SDM & $\begin{array}{l}\text { Not responsive to agonists ( } 4 \alpha \text { PDD, Hypotonicity } \\
\text { and AA), cause SMD Kozlowski type, and } \\
\text { Metatropic Dysplasia }\end{array}$ & Krakow et al.,2009 \\
\hline & \multirow[t]{2}{*}{ hTRPVA1 } & G955A & SDM & Slower inactivation rate. Lower rectification rates. & Benedikt et al.,2009 \\
\hline & & G958R & SDM & $\begin{array}{l}\text { Inward-rectifier, constitutively active at resting } \\
\text { potential, and impaired response to AITC }\end{array}$ & Benedikt et al.,2009 \\
\hline \multirow[t]{7}{*}{ W697 } & \multirow[t]{3}{*}{ rTRPV1 } & W697 & Structure & $\begin{array}{l}\text { It forms a hydrogen bond with the main chain } \\
\text { carbonyl oxygen of F559 at the beginning of the } \\
\text { S4-S5 linker }\end{array}$ & Liao et al.,2013 \\
\hline & & W697A & SDM & Low Response to Cap/Em & Valente et al., 2008 \\
\hline & & W697x & SDM & $\begin{array}{l}\text { Low Response to Cap/Em, Affect allosteric } \\
\text { activation }\end{array}$ & $\begin{array}{l}\text { Gregorio-Teruel et } \\
\text { al.,2014 }\end{array}$ \\
\hline & TRPV3 & W692G & SDM & Gain of Function, Olmsted Syndrome & Lin et al.,2012 \\
\hline & TRPV4 & W733R & SDM & $\begin{array}{l}\text { Gain of Function, limited agonist response, and } \\
\text { not inactivation to long depolarization }\end{array}$ & Teng et al.,2015 \\
\hline & TRPC3 & W673 & Structure & $\begin{array}{l}\text { It is extensively coupled with the S4-S5 linker } \\
\text { through interactions with G552 }\end{array}$ & Fan et al.,2018a \\
\hline & TRPC4 & W674 & Structure & $\begin{array}{l}\text { Coupled with the S4-S5 linker through } \\
\text { interactions with G553 and P546 on TM4 }\end{array}$ & Fan et al.,2018 \\
\hline
\end{tabular}


Table 3. Summary of mutation effects of residues from the AC or the conserved TM4 residue connecting TM4 with TM5 in the literature. First column indicates the equivalent signature residue in the rTRPV1 sequence. Second and third columns indicate the channel studied and the specific mutation introduced, respectively. Fourth column corresponds to the effect of the mutation and/or proposed function.

\begin{tabular}{|c|c|c|c|c|}
\hline $\begin{array}{l}\text { TRPV1 } \\
\text { Position }\end{array}$ & Channel & Mutation & Effect & Refference \\
\hline \multirow[t]{2}{*}{ F/Y444 } & TRPV1 & Y444S & Non-functional & Boukalova et al.,2013 \\
\hline & mTRPM3 & Y885T & $\begin{array}{l}\text { Impaired non-canonical current induced by } \\
\text { pregnenolone sulphate + clotrimazol }\end{array}$ & Held et al.,2018 \\
\hline \multirow[t]{2}{*}{ F448 } & TRPV1 & F448L & $\begin{array}{l}\text { Decreassed } \mathrm{pH} \text { response but mantain all Cap } \\
\text { responsivness }\end{array}$ & Boukalova et al.,2013 \\
\hline & mTRPM3 & Y888T & $\begin{array}{l}\text { Similar to } w t \text { response to pregnenolone } \\
\text { sulphate + clotrimazol }\end{array}$ & Held et al.,2018 \\
\hline \multirow[t]{6}{*}{ Y/F554 } & TRPV1 & Y554A & Non-fuctional (Cap $10 \mu \mathrm{M},-70$ to $200 \mathrm{mV}, 48^{\circ} \mathrm{C}$ ) & Boukalova et al.,2010 \\
\hline & & Y554F & Normal responsivness & Boukalova et al.,2010 \\
\hline & & Y554A & Not responsive to $\mathrm{pH}$, Cap and RTX & Elokely et al.,2015 \\
\hline & & Y554A & Increased sensitivity and affinity to 2 -APB & Singh et al.,2018 \\
\hline & TRPV2 & Y514A & Increased sensitivity and affinity to 2 -APB & Singh et al.,2018 \\
\hline & TRPV3 & Y564A & Increased affinity to $2-A P B$ & Singh et al.,2018 \\
\hline \multirow[t]{2}{*}{ Y/F555 } & TRPV1 & Y555S & Non-fuctional (Cap $10 \mu \mathrm{M},-70$ to $200 \mathrm{mV}, 48^{\circ} \mathrm{C}$ ) & Boukalova et al.,2010 \\
\hline & & Y555F & Normal responsivness & Boukalova et al.,2010 \\
\hline \multirow[t]{8}{*}{ W549 } & rTRPV1 & W549A & $\begin{array}{l}\text { Not responsive to Cap (and RTX and others) and } \\
\mathrm{pH}\end{array}$ & Ohbuchi et al.,2016 \\
\hline & rTRPV2 & W549A & $\begin{array}{l}\text { Interaction with vanillyl moiety of RTX or } \\
\text { Capsaicin }\end{array}$ & Gavva et al.,2004 \\
\hline & hTRPV4 & W568A & $\begin{array}{l}\text { Impaired responsivness to heat and agonists } \\
(4 \alpha-P D D \text { and BAA); but responsive to swelling } \\
\text { and endogen lipids }\end{array}$ & Vriens et al.,2007 \\
\hline & mTRPM3 & W982R & $\begin{array}{l}\text { Abolished non-canonical current induced by } \\
\text { pregnenolone sulphate + clotrimazol }\end{array}$ & Held et al.,2018 \\
\hline & mTRPM3 & W982F & $\begin{array}{l}\text { Similar to } w t \text { response to pregnenolone } \\
\text { sulphate + clotrimazol }\end{array}$ & Held et al.,2018 \\
\hline & hTRPA1 & Y840F & Reduced potency of ligand (GNE551) & Liu et al.,2021 \\
\hline & hTRPA1 & $\begin{array}{l}\text { Y840W/ } \\
\text { H/L/A }\end{array}$ & $\begin{array}{l}\text { Completely abolished potency of ligand } \\
\text { (GNE551) }\end{array}$ & Liu et al.,2021 \\
\hline & hTRPA1 & Y840A & $\begin{array}{l}\text { Impaired response to AITC and almost abolished } \\
\text { to } \beta \text {-Eudesmol }\end{array}$ & Ohara et al.,2015 \\
\hline
\end{tabular}




\section{REFERENCES LIST}

461

462

463

464

465

466

467

468

469

470

471

472

473

474

475

476

477

478

479

480

481

482

483

484

485

486

487

488

489

490

491

492

493

494

Altenhoff A.M., Train C.M., Gilbert K.J., Mediratta I., Mendes de Farias T., Moi D., Nevers Y., Radoykova H.S., Rossier V., Warwick Vesztrocy A., Glover N.M., Dessimoz C. 2021. “OMA orthology in 2021: website overhaul, conserved isoforms, ancestral gene order and more" Nucleic Acids Res. 49(D1):D373-D379. doi: 10.1093/nar/gkaa1007.

Altschul, S; Gish, W; Miller, W; Myers, E; Lipman, D. 1990. “Basic local alignment search tool” Journal of Molecular Biology 215 (3):403-410. https://doi.org/10.1016/S00222836(05)80360-2.

Arias-Darraz, Luis, Charlotte K Colenso, Luis A Veliz, Juan P Vivar, Sylvana Cardenas, and Sebastian Brauchi. 2015. "A TRP Conductance Modulates Repolarization after SensoryDependent Depolarization in Chlamydomonas Reinhardtii". Plant Signaling \& Behavior 10 (8): e1052924. https://doi.org/10.1080/15592324.2015.1052924.

Autzen, Henriette E., Alexander G. Myasnikov, Melody G. Campbell, Daniel Asarnow, David Julius, and Yifan Cheng. 2017. "Structure of the Human TRPM4 Ion Channel in a Lipid Nanodisc". Science 359 (6372): 228-32. https://doi.org/10.1126/science.aar4510.

Bandell, Michael, Adrienne E Dubin, Matt J Petrus, Anthony Orth, Jayanti Mathur, Sun Wook Hwang, and Ardem Patapoutian. 2006. "High-Throughput Random Mutagenesis Screen Reveals TRPM8 Residues Specifically Required for Activation by Menthol”. Nature Neuroscience 9 (4): 493-500. https://doi.org/10.1038/nn1665.

Beck, Andreas, Tilman Speicher, Christof Stoerger, Thomas Sell, Viviane Dettmer, Siti A. Jusoh, Ammar Abdulmughni, et al. 2013. "Conserved Gating Elements in TRPC4 and TRPC5 Channels”. Journal of Biological Chemistry 288 (27): 19471-83.

https://doi.org/10.1074/jbc.m113.478305.

Benedikt, Jan, Abdul Samad, Rudiger Ettrich, Jan Teisinger, and Viktorie Vlachova. 2009. "Essential Role for the Putative S6 Inner Pore Region in the Activation Gating of the Human TRPA1 Channel". Biochimica Et Biophysica Acta (BBA) - Molecular Cell Research 1793 (7): 1279-88. https://doi.org/10.1016/j.bbamcr.2009.04.014.

Bidaux, Gabriel, Miriam Sgobba, Loic Lemonnier, Anne-Sophie Borowiec, Lucile Noyer, Srdan Jovanovic, Alexander V. Zholos, and Shozeb Haider. 2015. "Functional and Modeling Studies of the Transmembrane Region of the TRPM8 Channel”. Biophysical Journal 109 (9): 1840-51. https://doi.org/10.1016/j.bpj.2015.09.027.

Boukalova, Stepana, Jan Teisinger, and Viktorie Vlachova. 2013. "Protons Stabilize the Closed Conformation of Gain-of-Function Mutants of the TRPV1 Channel”. Biochimica Et Biophysica Acta (BBA) - Molecular Cell Research 1833 (3): 520-28.

https://doi.org/10.1016/j.bbamcr.2012.11.017. 
Boukalova, Stepana, Lenka Marsakova, Jan Teisinger, and Viktorie Vlachova. 2010.

"Conserved Residues within the Putative S4S5 Region Serve Distinct Functions among

499 Brauchi, S., P. Orio, and R. Latorre. 2004. "Clues to Understanding Cold Sensation:

500 Thermodynamics and Electrophysiological Analysis of the Cold Receptor TRPM8".

501 Proceedings of the National Academy of Sciences 101 (43): 15494-99.

502 https://doi.org/10.1073/pnas.0406773101.

503 Brauchi, Sebastian E., and Brad S. Rothberg. 2020. "Gating and Calcium-Sensing

504 Mechanisms of TRPA1 Channels Revealed". Cell Calcium 91 (November): 102278.

505 https://doi.org/10.1016/j.ceca.2020.102278.

506 Cao, Erhu, Maofu Liao, Yifan Cheng, and David Julius. 2013. "TRPV1 Structures in Distinct

507 Conformations Reveal Activation Mechanisms". Nature 504 (7478): 113-18.

508 https://doi.org/10.1038/nature12823.

509 Cao, Erhu. 2020. "Structural Mechanisms of Transient Receptor Potential Ion Channels".

510 Journal of General Physiology 152 (3). https://doi.org/10.1085/jgp.201811998.

511 Carvalho-de-Souza, Joao L., and Francisco Bezanilla. 2019. "Noncanonical Mechanism of

512 Voltage Sensor Coupling to Pore Revealed by Tandem Dimers of Shaker". Nature

513 Communications 10 (1). https://doi.org/10.1038/s41467-019-11545-7.

514 Castillo, Karen, Ignacio Diaz-Franulic, Jonathan Canan, Fernando Gonzalez-Nilo, and Ramon

515 Latorre. 2018. “Thermally Activated TRP Channels: Molecular Sensors for Temperature

516 Detection". Physical Biology 15 (2): 021001. https://doi.org/10.1088/1478-3975/aa9a6f.

517 Chandrabalan, Arundhasa, Martin J. McPhillie, Alyn H. Morice, Andrew N. Boa, and Laura R.

518 Sadofsky. 2019. "N-Cinnamoylanthranilates as Human TRPA1 Modulators: Structure-

519 Activity Relationships and Channel Binding Sites". European Journal of Medicinal Chemistry

520170 (May): 141-56. https://doi.org/10.1016/j.ejmech.2019.02.074.

521 Cheng, Yifan. 2018. "Single-Particle Cryo-EMHow Did It Get Here and Where Will It Go".

522 Science 361 (6405): 876-80. https://doi.org/10.1126/science.aat4346.

523 Chugunov, Anton O., Pavel E. Volynsky, Nikolay A. Krylov, Dmitry E. Nolde, and Roman G.

524 Efremov. 2016. "Temperature-Sensitive Gating of TRPV1 Channel as Probed by Atomistic

525 Simulations of Its Trans- and Juxtamembrane Domains". Scientific Reports 6 (1).

526 https://doi.org/10.1038/srep33112.

527 Clapham, David E. 2003. “TRP Channels as Cellular Sensors”. Nature 426 (6966): 517-24.

528 https://doi.org/10.1038/nature02196. 
529 Clapham, David E., and David L. Garbers. 2005. “International Union of Pharmacology. L.

530 Nomenclature and Structure-Function Relationships of CatSper and Two-Pore Channels".

531 Pharmacological Reviews 57 (4): 451-54. https://doi.org/10.1124/pr.57.4.7.

532 Crooks, G. E. 2004. “WebLogo: A Sequence Logo Generator”. Genome Research 14 (6): 1188-

533 90. https://doi.org/10.1101/gr.849004.

534 Duan, Jingjing, Zongli Li, Jian Li, Ana Santa-Cruz, Silvia Sanchez-Martinez, Jin Zhang, and 535 David E. Clapham. 2018. "Structure of Full-Length Human TRPM4". Proceedings of the 536 National Academy of Sciences 115 (10): 2377-82.

537 https://doi.org/10.1073/pnas.1722038115.

538 Duchatelet, Sabine, Solenn Pruvost, Simon de Veer, Sylvie Fraitag, Patrick Nitschké, 539 Christine Bole-Feysot, Christine Bodemer, and Alain Hovnanian. 2014. "A New TRPV3 540 Missense Mutation in a Patient With Olmsted Syndrome and Erythromelalgia". JAMA 541 Dermatology 150 (3): 303. https://doi.org/10.1001/jamadermatol.2013.8709.

542 Duo, Lina, Linghan Hu, Naxi Tian, Gen Cheng, Huijun Wang, Zhimiao Lin, Yun Wang, and 543 Yong Yang. 2018. "TRPV1 Gain-of-Function Mutation Impairs Pain and Itch Sensations in 544 Mice”. Molecular Pain 14 (January): 174480691876203.

545 https://doi.org/10.1177/1744806918762031.

546 Ekeberg, Magnus, Cecilia Lövkvist, Yueheng Lan, Martin Weigt, and Erik Aurell. 2013.

547 "Improved Contact Prediction in Proteins: Using Pseudolikelihoods to Infer Potts Models".

548 Physical Review E 87 (1). https://doi.org/10.1103/physreve.87.012707.

549 Elokely, Khaled, Phanindra Velisetty, Lucie Delemotte, Eugene Palovcak, Michael L. Klein, 550 Tibor Rohacs, and Vincenzo Carnevale. 2015. "Understanding TRPV1 Activation by Ligands:

551 Insights from the Binding Modes of Capsaicin and Resiniferatoxin". Proceedings of the

$552 \quad$ National Academy of Sciences 113 (2): E137-E145.

553 https://doi.org/10.1073/pnas.1517288113.

554 Elokely, Khaled, Phanindra Velisetty, Lucie Delemotte, Eugene Palovcak, Michael L. Klein, 555 Tibor Rohacs, and Vincenzo Carnevale. 2015. "Understanding TRPV1 Activation by Ligands:

556 Insights from the Binding Modes of Capsaicin and Resiniferatoxin". Proceedings of the

$557 \quad$ National Academy of Sciences 113 (2): E137-E145.

558 https://doi.org/10.1073/pnas.1517288113.

559 Fan, Chen, Wooyoung Choi, Juan Du, and Wei Lü. 2018. "Structure of the Human Lipid560 Sensitive Cation Channel TRPC3”, March. https://doi.org/10.1101/286153.

561 Fan, Chen, Wooyoung Choi, Weinan Sun, Juan Du, and Wei Lü. 2018. "Structure of the 562 Human Lipid-Gated Cation Channel TRPC3". ELife 7 (May).

563 https://doi.org/10.7554/elife.36852.

564 Galione, A. 2010. "NAADP Receptors". Cold Spring Harbor Perspectives in Biology 3 (1):

565 a004036-a004036. https://doi.org/10.1101/cshperspect.a004036. 
566 Gao, Yuan, Erhu Cao, David Julius, and Yifan Cheng. 2016. "TRPV1 Structures in Nanodiscs

567 Reveal Mechanisms of Ligand and Lipid Action”. Nature 534 (7607): 347-51.

568 https://doi.org/10.1038/nature17964.

569 Garcia-Elias, Anna, Alejandro Berna-Erro, Fanny Rubio-Moscardo, Carlos Pardo-Pastor, 570 Sanela Mrkonjić, Romina V. Sepúlveda, Rubén Vicente, Fernando González-Nilo, and 571 Miguel A. Valverde. 2015. "Interaction between the Linker Pre-S1, and TRP Domains 572 Determines Folding, Assembly, and Trafficking of TRPV Channels". Structure 23 (8): 1404573 13. https://doi.org/10.1016/j.str.2015.05.018.

574 Gavva, Narender R., Lana Klionsky, Yusheng Qu, Licheng Shi, Rami Tamir, Steve Edenson, 575 T.J. Zhang, et al. 2004. "Molecular Determinants of Vanilloid Sensitivity in TRPV1". Journal 576 of Biological Chemistry 279 (19): 20283-95. https://doi.org/10.1074/jbc.m312577200.

577 Grandl, Jörg, Hongzhen Hu, Michael Bandell, Badry Bursulaya, Manuela Schmidt, Matt 578 Petrus, and Ardem Patapoutian. 2008. "Pore Region of TRPV3 Ion Channel Is Specifically Required for Heat Activation”. Nature Neuroscience 11 (9): 1007-13.

580 https://doi.org/10.1038/nn.2169.

581 Gregorio-Teruel, Lucia, Pierluigi Valente, José Manuel González-Ros, Gregorio Fernández582 Ballester, and Antonio Ferrer-Montiel. 2014. "Mutation of I696 and W697 in the TRP Box of 583 Vanilloid Receptor Subtype I Modulates Allosteric Channel Activation”. Journal of General 584 Physiology 143 (3): 361-75. https://doi.org/10.1085/jgp.201311070.

585 Guo, Jiangtao, Ji She, Weizhong Zeng, Qingfeng Chen, Xiao-chen Bai, and Youxing Jiang. 586 2017. "Structures of the Calcium-Activated Non-Selective Cation Channel TRPM4". Nature 587552 (7684): 205-9. https://doi.org/10.1038/nature24997.

588 Held, Katharina, Fabian Gruss, Vincenzo Davide Aloi, Annelies Janssens, Chris Ulens, Thomas Voets, and Joris Vriens. 2018. "Mutations in the Voltage-Sensing Domain Affect the Alternative Ion Permeation Pathway in the TRPM3 Channel”. The Journal of Physiology 596 (12): 2413-32. https://doi.org/10.1113/jp274124.

593

Hilton, Jacob K., Minjoo Kim, and Wade D. Van Horn. 2019. “Structural and Evolutionary Insights Point to Allosteric Regulation of TRP Ion Channels". Accounts of Chemical Research 52 (6): 1643-52. https://doi.org/10.1021/acs.accounts.9b00075.

595 Himmel, Nathaniel J., and Daniel N. Cox. 2020. “Transient Receptor Potential Channels: Current Perspectives on Evolution Structure, Function and Nomenclature". Proceedings of 598 https://doi.org/10.1098/rspb.2020.1309.

599 Hofmann, Laura, Hongmei Wang, Wang Zheng, Stephan E. Philipp, Patricia Hidalgo, Adolfo 600 Cavalié, Xing-Zhen Chen, Andreas Beck, and Veit Flockerzi. 2017. "The S4S5 Linker Gearbox 601 of TRP Channel Gating". Cell Calcium 67 (November): 156-65.

602 https://doi.org/10.1016/j.ceca.2017.04.002. 
603 Huffer, Katherine E., Antoniya A. Aleksandrova, Andrés Jara-Oseguera, Lucy R. Forrest, and

604 Kenton J. Swartz. 2020. "Global Alignment and Assessment of TRP Channel

605 Transmembrane Domain Structures to Explore Functional Mechanisms”, May.

606 https://doi.org/10.1101/2020.05.14.096792.

607 Hughes, Taylor E. T., Ruth A. Pumroy, Aysenur Torun Yazici, Marina A. Kasimova, Edwin C. 608 Fluck, Kevin W. Huynh, Amrita Samanta, et al. 2018. "Structural Insights on TRPV5 Gating 609 by Endogenous Modulators". Nature Communications 9 (1).

610 https://doi.org/10.1038/s41467-018-06753-6.

611 Hui, Kwokyin, Beiying Liu, and Feng Qin. 2003. “Capsaicin Activation of the Pain Receptor 612 VR1: Multiple Open States from Both Partial and Full Binding”. Biophysical Journal 84 (5): 613 2957-68. https://doi.org/10.1016/s0006-3495(03)70022-8.

614 Humphrey, William, Andrew Dalke, and Klaus Schulten. 1996. "VMD: Visual Molecular 615 Dynamics". Journal of Molecular Graphics 14 (1): 33-38. https://doi.org/10.1016/0263$6167855(96) 00018-5$.

617 Kadowaki, Tatsuhiko. 2015. “Evolutionary Dynamics of Metazoan TRP Channels”. Pflügers 618 Archiv - European Journal of Physiology 467 (10): 2043-53.

619 https://doi.org/10.1007/s00424-015-1705-5.

620 Kasimova, M.A., D. Granata, and V. Carnevale. 2016. “Voltage-Gated Sodium Channels”. In 621 Na Channels from Phyla to Function, 261-86. Elsevier.

622 https://doi.org/10.1016/bs.ctm.2016.05.002.

623 Kasimova, Marina A., Aysenur Torun Yazici, Yevgen Yudin, Daniele Granata, Michael L. 624 Klein, Tibor Rohacs, and Vincenzo Carnevale. 2018. "A Consistent Picture of TRPV1 625 Activation Emerges from Molecular Simulations and Experiments", April.

626 https://doi.org/10.1101/310151.

627 Kasimova, Marina A., Aysenur Yazici, Yevgen Yudin, Daniele Granata, Michael L. Klein, Tibor 628 Rohacs, and Vincenzo Carnevale. 2017. "TRPV1 Activation Relies on

629 Hydration/Dehydration of Nonpolar Cavities", March. https://doi.org/10.1101/114546.

630 Kasimova, Marina A., Aysenur Yazici, Yevgen Yudin, Daniele Granata, Michael L. Klein, Tibor 631 Rohacs, and Vincenzo Carnevale. 2018. "Ion Channel Sensing: Are Fluctuations the Crux of 632 the Matter?". The Journal of Physical Chemistry Letters 9 (6): 1260-64.

633 https://doi.org/10.1021/acs.jpclett.7b03396.

634 Katoh, Kazutaka, John Rozewicki, and Kazunori D Yamada. 2017. "MAFFT Online Service: 635 Multiple Sequence Alignment Interactive Sequence Choice and Visualization". Briefings in 636 Bioinformatics 20 (4): 1160-66. https://doi.org/10.1093/bib/bbx108.

637 Kim, Minjoo, Nicholas J. Sisco, Jacob K. Hilton, Camila M. Montano, Manuel A. Castro, Brian 638 R. Cherry, Marcia Levitus, and Wade D. Van Horn. 2020. "Evidence That the TRPV1 S1-S4 
639 Membrane Domain Contributes to Thermosensing". Nature Communications 11 (1).

640 https://doi.org/10.1038/s41467-020-18026-2.

641 Kintzer, Alexander F., and Robert M. Stroud. 2017. "On the Structure and Mechanism of

642 Two-Pore Channels". The FEBS Journal 285 (2): 233-43.

643 https://doi.org/10.1111/febs.14154.

644 Kitaguchi, Tetsuya, and Kenton J. Swartz. 2005. “An Inhibitor of TRPV1 Channels Isolated 645 from Funnel Web Spider Venom”. Biochemistry 44 (47): 15544-49.

646 https://doi.org/10.1021/bi0514941.

647 Klausen, Thomas Kjær, Annelies Janssens, Jean Prenen, Grzegorz Owsianik, Else Kay 648 Hoffmann, Stine Falsig Pedersen, and Bernd Nilius. 2014. "Single Point Mutations of 649 Aromatic Residues in Transmembrane Helices 5 and -6 Differentially Affect TRPV4 650 Activation by $\alpha$-PDD and Hypotonicity: Implications for the Role of the Pore Region in 651 Regulating TRPV4 Activity". Cell Calcium 55 (1): 38-47.

652 https://doi.org/10.1016/j.ceca.2013.11.001.

653 Krakow, Deborah, Joris Vriens, Natalia Camacho, Phi Luong, Hannah Deixler, Tara L. Funari, 654 Carlos A. Bacino, et al. 2009. "Mutations in the Gene Encoding the Calcium-Permeable Ion 655 Channel TRPV4 Produce Spondylometaphyseal Dysplasia Kozlowski Type and Metatropic 656 Dysplasia". The American Journal of Human Genetics 84 (3): 307-15.

657 https://doi.org/10.1016/j.ajhg.2009.01.021.

658 Kuzhikandathil, Eldo V., Haibin Wang, Tamas Szabo, Natasha Morozova, Peter M. Blumberg, 659 and Gerry S. Oxford. 2001. "Functional Analysis of Capsaicin Receptor (Vanilloid Receptor 660 Subtype 1) Multimerization and Agonist Responsiveness Using a Dominant Negative 661 Mutation". The Journal of Neuroscience 21 (22): 8697-8706.

662 https://doi.org/10.1523/jneurosci.21-22-08697.2001.

663 Lainez, Sergio, Karl Peter Schlingmann, Jenny van der Wijst, Bernd Dworniczak, Femke van 664 Zeeland, Martin Konrad, René J Bindels, and Joost G Hoenderop. 2013. "New TRPM6 665 Missense Mutations Linked to Hypomagnesemia with Secondary Hypocalcemia". European 666 Journal of Human Genetics 22 (4): 497-504. https://doi.org/10.1038/ejhg.2013.178.

667 Latorre, Ramon, Cristián Zaelzer, and Sebastian Brauchi. 2009. "StructureFunctional 668 Intimacies of Transient Receptor Potential Channels". Quarterly Reviews of Biophysics 42 669 (3): 201-46. https://doi.org/10.1017/s0033583509990072.

670 Liao, Maofu, Erhu Cao, David Julius, and Yifan Cheng. 2013. "Structure of the TRPV1 Ion 671 Channel Determined by Electron Cryo-Microscopy”. Nature 504 (7478): 107-12.

672 https://doi.org/10.1038/nature12822.

673 Lin, Zhimiao, Quan Chen, Mingyang Lee, Xu Cao, Jie Zhang, Donglai Ma, Long Chen, et al. 674 2012. "Exome Sequencing Reveals Mutations in TRPV3 as a Cause of Olmsted Syndrome". 
675 The American Journal of Human Genetics 90 (3): 558-64.

676 https://doi.org/10.1016/j.ajhg.2012.02.006.

677 Liu, Beiying, Jing Yao, Yingwei Wang, Hui Li, and Feng Qin. 2009. “Proton Inhibition of 678 Unitary Currents of Vanilloid Receptors”. Journal of General Physiology 134 (3): 243-58.

679 https://doi.org/10.1085/jgp.200910255.

680 Liu, Chang, Rebecca Reese, Simon Vu, Lionel Rougé, Shannon D. Shields, Satoko KakiuchiKiyota, Huifen Chen, et al. 2021. "A Non-Covalent Ligand Reveals Biased Agonism of the TRPA1 Ion Channel”. Neuron 109 (2): 273-84.e4. https://doi.org/10.1016/j.neuron.2020.10.014.

Malkia, Annika, María Pertusa, Gregorio Fernández-Ballester, Antonio Ferrer-Montiel, and Félix Viana. 2009. "Differential Role of the Menthol-Binding Residue Y745 in the Antagonism of Thermally Gated TRPM8 Channels”. Molecular Pain 5 (January): 1744-

688 McGoldrick, Luke L., Appu K. Singh, Lusine Demirkhanyan, Ting-Yu Lin, Ryan G. Casner, 689 Eleonora Zakharian, and Alexander I. Sobolevsky. 2019. "Structure of the Thermo-Sensitive TRP Channel TRP1 from the Alga Chlamydomonas Reinhardtii". Nature Communications 10 (1). https://doi.org/10.1038/s41467-019-12121-9.

Minh, Bui Quang, Heiko A Schmidt, Olga Chernomor, Dominik Schrempf, Michael D Woodhams, Arndt von Haeseler, and Robert Lanfear. 2020. "IQ-TREE 2: New Models and Efficient Methods for Phylogenetic Inference in the Genomic Era”. Edited by Emma Teeling. 695 Molecular Biology and Evolution 37 (5): 1530-34.

696 https://doi.org/10.1093/molbev/msaa015.

697 Munns, Clare H., Man-Kyo Chung, Yuly E. Sanchez, L. Mario Amzel, and Michael J. Caterina. 698 2015. "Role of the Outer Pore Domain in Transient Receptor Potential Vanilloid 1 Dynamic 699 Permeability to Large Cations”. Journal of Biological Chemistry 290 (9): 5707-24. https://doi.org/10.1074/jbc.m114.597435.

701 Nadezhdin, Kirill D., Arthur Neuberger, Yuri A. Trofimov, Nikolay A. Krylov, Viktor Sinica, Nikita Kupko, Viktorie Vlachova, Eleonora Zakharian, Roman G. Efremov, and Alexander I. Sobolevsky. 2021. "Structural Mechanism of Heat-Induced Opening of a Temperature-

704 Sensitive TRP Channel”. Nature Structural \& Molecular Biology 28 (7): 564-72.

705 https://doi.org/10.1038/s41594-021-00615-4.

706 Nadezhdin, Kirill D., Arthur Neuberger, Yury A. Nikolaev, Lyle A. Murphy, Elena O.

707 Gracheva, Sviatoslav N. Bagriantsev, and Alexander I. Sobolevsky. 2021. "Extracellular Cap Domain Is an Essential Component of the TRPV1 Gating Mechanism". Nature

709 Communications 12 (1). https://doi.org/10.1038/s41467-021-22507-3.

710 Neale, E. J., D. J. S. Elliott, M. Hunter, and A. Sivaprasadarao. 2003. "Evidence for 711 Intersubunit Interactions between S4 and S5 Transmembrane Segments of the Shaker 
712 Potassium Channel”. Journal of Biological Chemistry 278 (31): 29079-85.

713 https://doi.org/10.1074/jbc.m301991200/6493.

714 Nilius, Bernd, Grzegorz Owsianik, Thomas Voets, and John A. Peters. 2007. “Transient

715 Receptor Potential Cation Channels in Disease”. Physiological Reviews 87 (1): 165-217.

716 https://doi.org/10.1152/physrev.00021.2006.

717 Ohara, Kazuaki, Takafumi Fukuda, Hiroyuki Okada, Sayoko Kitao, Yuko Ishida, Kyoko Kato,

718 Chika Takahashi, Mikio Katayama, Kunitoshi Uchida, and Makoto Tominaga. 2015.

719

"Identification of Significant Amino Acids in Multiple Transmembrane Domains of Human

720

Transient Receptor Potential Ankyrin 1 (TRPA1) for Activation by Eudesmol an Oxygenized

Sesquiterpene in Hop Essential Oil”. Journal of Biological Chemistry 290 (5): 3161-71.

722

https://doi.org/10.1074/jbc.m114.600932.

723 Ohbuchi, Katsuya, Yoshikazu Mori, Kazuo Ogawa, Eiji Warabi, Masahiro Yamamoto, and

724 Takatsugu Hirokawa. 2016. "Detailed Analysis of the Binding Mode of Vanilloids to

725 Transient Receptor Potential Vanilloid Type I (TRPV1) by a Mutational and Computational

726

727

Study". Edited by Andrej A. Romanovsky. PLOS ONE 11 (9): e0162543.

https://doi.org/10.1371/journal.pone.0162543.

728 Owsianik, Grzegorz, Karel Talavera, Thomas Voets, and Bernd Nilius. 2006. "PERMEATION

729 AND SELECTIVITY OF TRP CHANNELS”. Annual Review of Physiology 68 (1): 685-717.

730 https://doi.org/10.1146/annurev.physiol.68.040204.101406.

731 Palovcak, Eugene, Lucie Delemotte, Michael L. Klein, and Vincenzo Carnevale. 2015.

732 "Comparative Sequence Analysis Suggests a Conserved Gating Mechanism for TRP

733 Channels". Journal of General Physiology 146 (1): 37-50.

734 https://doi.org/10.1085/jgp.201411329.

735 Paulsen, Candice E., Jean-Paul Armache, Yuan Gao, Yifan Cheng, and David Julius. 2015.

736 "Structure of the TRPA1 Ion Channel Suggests Regulatory Mechanisms". Nature 520

737 (7548): 511-17. https://doi.org/10.1038/nature14367.

738 Payandeh, Jian, Todd Scheuer, Ning Zheng, and William A. Catterall. 2011. “The Crystal

Structure of a Voltage-Gated Sodium Channel". Nature 475 (7356): 353-58.

740 https://doi.org/10.1038/nature10238.

741 Peng, Guangda, Xiao Shi, and Tatsuhiko Kadowaki. 2015. "Evolution of TRP Channels

742 Inferred by Their Classification in Diverse Animal Species". Molecular Phylogenetics and

743 Evolution 84 (March): 145-57. https://doi.org/10.1016/j.ympev.2014.06.016.

744 Penny, Christopher J., Taufiq Rahman, Altin Sula, Andrew J. Miles, B. A. Wallace, and Sandip 745 Patel. 2016. "Isolated Pores Dissected from Human Two-Pore Channel 2 Are Functional".

746 Scientific Reports 6 (1). https://doi.org/10.1038/srep38426. 
Piovesan, Damiano, Giovanni Minervini, and Silvio C.E. Tosatto. 2016. "The RING 2.0 Web Server for High Quality Residue Interaction Networks". Nucleic Acids Research 44 (W1): W367-W374. https://doi.org/10.1093/nar/gkw315.

750 Poblete, Horacio, Ingrid Oyarzún, Pablo Olivero, Jeffrey Comer, Matías Zuñiga, Romina V.

751

752

753 Sepulveda, David Báez-Nieto, Carlos González Leon, Fernando González-Nilo, and Ramón Latorre. 2014. "Molecular Determinants of Phosphatidylinositol 4,5-Bisphosphate (PI(4,5)P2) Binding to Transient Receptor Potential V1 (TRPV1) Channels". Journal of Biological Chemistry 290 (4): 2086-98. https://doi.org/10.1074/jbc.m114.613620. CHANNELS". Annual Review of Physiology 68 (1): 619-47.

758 Romero-Romero, Sergio, Froylan Gomez Lagunas, and Daniel Balleza. 2017. "Side Chain Flexibility and Coupling between the S4-S5 Linker and the TRP Domain in ThermoSensitive TRP Channels: Insights from Protein Modeling". Proteins: Structure Function, and Bioinformatics 85 (4): 630-46. https://doi.org/10.1002/prot.25243. Microscopy of TRP Channels". In Methods in Molecular Biology, 39-50. Springer New York. https://doi.org/10.1007/978-1-4939-9446-5_3.

Shem-Ad, Tzilhav, Orr Irit, and Ofer Yifrach. 2013. "Inter-Subunit Interactions across the Upper Voltage Sensing-Pore Domain Interface Contribute to the Concerted Pore Opening Transition of Kv Channels". Edited by Bernard Attali. PLoS ONE 8 (12): e82253. https://doi.org/10.1371/journal.pone.0082253.

769 Sierra-Valdez, Francisco, Caleigh M. Azumaya, Luis O. Romero, Terunaga Nakagawa, and Julio F. Cordero-Morales. 2018. "StructureFunction Analyses of the Ion Channel TRPC3 Reveal That Its Cytoplasmic Domain Allosterically Modulates Channel Gating”. Journal of

773 Singh, Appu K., Kei Saotome, Luke L. McGoldrick, and Alexander I. Sobolevsky. 2018. "Structural Bases of TRP Channel TRPV6 Allosteric Modulation by 2-APB". Nature Communications 9 (1). https://doi.org/10.1038/s41467-018-04828-y.

776 Singh, Appu K., Luke L. McGoldrick, and Alexander I. Sobolevsky. 2018. "Structure and Gating Mechanism of the Transient Receptor Potential Channel TRPV3". Nature Structural \& Molecular Biology 25 (9): 805-13. https://doi.org/10.1038/s41594-018-0108-7.

Steinberg, Ximena, Carolyne Lespay-Rebolledo, and Sebastian Brauchi. 2014. "A Structural View of Ligand-Dependent Activation in ThermoTRP Channels". Frontiers in Physiology 5

782 Strübing, Carsten, Grigory Krapivinsky, Luba Krapivinsky, and David E. Clapham. 2003. 783 "Formation of Novel TRPC Channels by Complex Subunit Interactions in Embryonic Brain". 
784 Journal of Biological Chemistry 278 (40): 39014-19.

785 https://doi.org/10.1074/jbc.m306705200.

786 Susankova, K., R. Ettrich, L. Vyklicky, J. Teisinger, and V. Vlachova. 2007. “Contribution of the Putative Inner-Pore Region to the Gating of the Transient Receptor Potential Vanilloid Subtype 1 Channel (TRPV1)". Journal of Neuroscience 27 (28): 7578-85. https://doi.org/10.1523/jneurosci.1956-07.2007.

790 Taberner, Francisco José, Ainara López-Córdoba, Gregorio Fernández-Ballester, Yuri 791 Korchev, and Antonio Ferrer-Montiel. 2014. "The Region Adjacent to the C-End of the Inner 792 Gate in Transient Receptor Potential Melastatin 8 (TRPM8) Channels Plays a Central Role in 793 Allosteric Channel Activation". Journal of Biological Chemistry 289 (41): 28579-94.

794 https://doi.org/10.1074/jbc.m114.577478.

795 Tang, Qinglin, Wenjun Guo, Li Zheng, Jing-Xiang Wu, Meng Liu, Xindi Zhou, Xiaolin Zhang, 796 and Lei Chen. 2018. "Structure of the Receptor-Activated Human TRPC6 and TRPC3 Ion 797 Channels". Cell Research 28 (7): 746-55. https://doi.org/10.1038/s41422-018-0038-2.

798 Teng, Jinfeng, Stephen H. Loukin, Andriy Anishkin, and Ching Kung. 2015. “L596W733 Bond 799 between the Start of the S4S5 Linker and the TRP Box Stabilizes the Closed State of TRPV4 800 Channel". Proceedings of the National Academy of Sciences 112 (11): 3386-91.

801 https://doi.org/10.1073/pnas.1502366112.

802 Valente, Pierluigi, Nuria García-Sanz, Ana Gomis, Asia Fernández-Carvajal, Gregorio 803 Fernández-Ballester, Félix Viana, Carlos Belmonte, and Antonio Ferrer-Montiel. 2008. 804 "Identification of Molecular Determinants of Channel Gating in the Transient Receptor 805 Potential Box of Vanilloid Receptor I". The FASEB Journal 22 (9): 3298-3309.

806 https://doi.org/10.1096/fj.08-107425.

807 Venkatachalam, Kartik, and Craig Montell. 2007. “TRP Channels”. Annual Review of 808 Biochemistry 76 (1): 387-417.

809 https://doi.org/10.1146/annurev.biochem.75.103004.142819.

810 Vinayagam, Deivanayagabarathy, Dennis Quentin, Oleg Sitsel, Felipe Merino, Markus

811 Stabrin, Oliver Hofnagel, Maolin Yu, Mark W. Ledeboer, Goran Malojcic, and Stefan Raunser. 812 2020. "Structural Basis of TRPC4 Regulation by Calmodulin and Pharmacological Agents",

813 July. https://doi.org/10.1101/2020.06.30.180778.

814 Vinayagam, Deivanayagabarathy, Thomas Mager, Amir Apelbaum, Arne Bothe, Felipe 815 Merino, Oliver Hofnagel, Christos Gatsogiannis, and Stefan Raunser. 2018. "Electron Cryo816 Microscopy Structure of the Canonical TRPC4 Ion Channel”. ELife 7 (May).

817 https://doi.org/10.7554/elife.36615.

818 Voolstra, Olaf, and Armin Huber. 2014. "Post-Translational Modifications of TRP Channels". 819 Cells 3 (2): 258-87. https://doi.org/10.3390/cells3020258. 
820 Vriens, Joris, Grzegorz Owsianik, Annelies Janssens, Thomas Voets, and Bernd Nilius. 2007. 821 "Determinants of $4 \$ \backslash$ Upalpha \$-Phorbol Sensitivity in Transmembrane Domains 3 and 4 of 822 the Cation Channel TRPV4". Journal of Biological Chemistry 282 (17): 12796-803.

823 https://doi.org/10.1074/jbc.m610485200.

824 Waterhouse, A. M., J. B. Procter, D. M. A. Martin, M. Clamp, and G. J. Barton. 2009. “Jalview 825 Version 2-a Multiple Sequence Alignment Editor and Analysis Workbench". Bioinformatics 82625 (9): 1189-91. https://doi.org/10.1093/bioinformatics/btp033.

827 Xiao, Rui, Jinbin Tian, Jisen Tang, and Michael X. Zhu. 2008. "The TRPV3 Mutation 828 Associated with the Hairless Phenotype in Rodents Is Constitutively Active". Cell Calcium 43 829 (4): 334-43. https://doi.org/10.1016/j.ceca.2007.06.004.

830 Yang, Fan, and Jie Zheng. 2017. “Understand Spiciness: Mechanism of TRPV1 Channel 831 Activation by Capsaicin”. Protein \& Cell 8 (3): 169-77. https://doi.org/10.1007/s13238832 016-0353-7.

833 Yang, Fan, Lizhen Xu, Bo Hyun Lee, Xian Xiao, Vladimir Yarov-Yarovoy, and Jie Zheng. 2020. 834 "An Unorthodox Mechanism Underlying Voltage Sensitivity of TRPV1 Ion Channel". 835 Advanced Science 7 (20): 2000575. https://doi.org/10.1002/advs.202000575.

836 Yang, Fan, Xian Xiao, Bo Hyun Lee, Simon Vu, Wei Yang, Vladimir Yarov-Yarovoy, and Jie 837 Zheng. 2018. "The Conformational Wave in Capsaicin Activation of Transient Receptor 838 Potential Vanilloid 1 Ion Channel”. Nature Communications 9 (1).

839 https://doi.org/10.1038/s41467-018-05339-6.

840 Yazici, Aysenur Torun, Eleonora Gianti, Marina A. Kasimova, Bo-Hyun Lee, Vincenzo 841 Carnevale, and Tibor Rohacs. 2021. "Dual Regulation of TRPV1 Channels by 842 Phosphatidylinositol via Functionally Distinct Binding Sites”. Journal of Biological Chemistry 843296 (January): 100573. https://doi.org/10.1016/j.jbc.2021.100573.

844 Yin, Ying, Mengyu Wu, Lejla Zubcevic, William F. Borschel, Gabriel C. Lander, and Seok-Yong 845 Lee. 2017. "Structure of the Cold- and Menthol-Sensing Ion Channel TRPM8". Science 359 846 (6372): 237-41. https://doi.org/10.1126/science.aan4325.

847 Yin, Ying, Son C. Le, Allen L. Hsu, Mario J. Borgnia, Huanghe Yang, and Seok-Yong Lee. 2019. 848 "Structural Basis of Cooling Agent and Lipid Sensing by the Cold-Activated TRPM8 849 Channel”. Science 363 (6430): eaav9334. https://doi.org/10.1126/science.aav9334.

850 Yu, Frank H., Vladimir Yarov-Yarovoy, George A. Gutman, and William A. Catterall. 2005. 851 "Overview of Molecular Relationships in the Voltage-Gated Ion Channel Superfamily".

852 Pharmacological Reviews 57 (4): 387-95. https://doi.org/10.1124/pr.57.4.13.

853 Yuan, Peng. 2019. "Structural Biology of ThermoTRPV Channels". Cell Calcium 84

854 (December): 102106. https://doi.org/10.1016/j.ceca.2019.102106. 
855 Zhang, Feng, Kenton Jon Swartz, and Andres Jara-Oseguera. 2019. “Conserved Allosteric 856 Pathways for Activation of TRPV3 Revealed through Engineering Vanilloid-Sensitivity". 857 ELife 8 (January). https://doi.org/10.7554/elife.42756.

858 Zhao, Jianhua, John V. Lin King, Candice E. Paulsen, Yifan Cheng, and David Julius. 2020. 859 "Irritant-Evoked Activation and Calcium Modulation of the TRPA1 Receptor". Nature 585

860 (7823): 141-45. https://doi.org/10.1038/s41586-020-2480-9.

861 Zheng, Wang, Ruikun Hu, Ruiqi Cai, Laura Hofmann, Qiaolin Hu, Mohammad Fatehi, 862 Wentong Long, et al. 2018. "Identification and Characterization of Hydrophobic Gate 863 Residues in TRP Channels". The FASEB Journal 32 (2): 639-53.

864 https://doi.org/10.1096/fj.201700599rr.

865 Zimova, Lucie, Viktor Sinica, Anna Kadkova, Lenka Vyklicka, Vlastimil Zima, Ivan Barvik, 866 and Viktorie Vlachova. 2018. "Intracellular Cavity of Sensor Domain Controls Allosteric 867 Gating of TRPA1 Channel”. Science Signaling 11 (514): eaan8621.

868 https://doi.org/10.1126/scisignal.aan8621.

869 Zubcevic, Lejla, William F Borschel, Allen L Hsu, Mario J Borgnia, and Seok-Yong Lee. 2019. 870 "Regulatory Switch at the Cytoplasmic Interface Controls TRPV Channel Gating". ELife 8 871 (May). https://doi.org/10.7554/elife.47746.

872 Zubcevic, Lejla. 2020. “TRP Channels Conformational Flexibility, and the Lipid Membrane”. 873 The Journal of Membrane Biology 253 (4): 299-308. https://doi.org/10.1007/s00232-020874 00127-0. 
bioBxiv preprint doi: https://doi.org/10.1101/2021.09.06.459056; this ve sion posted September 6,2021. The copyright holder for this preprint (Whicbuas not certified by peer review) is the author/funder, who has $\mathrm{g}$ anted bioRxiva license to display the preprint in perpetuity. It is made available under aCC-BY .0 International license. 1.

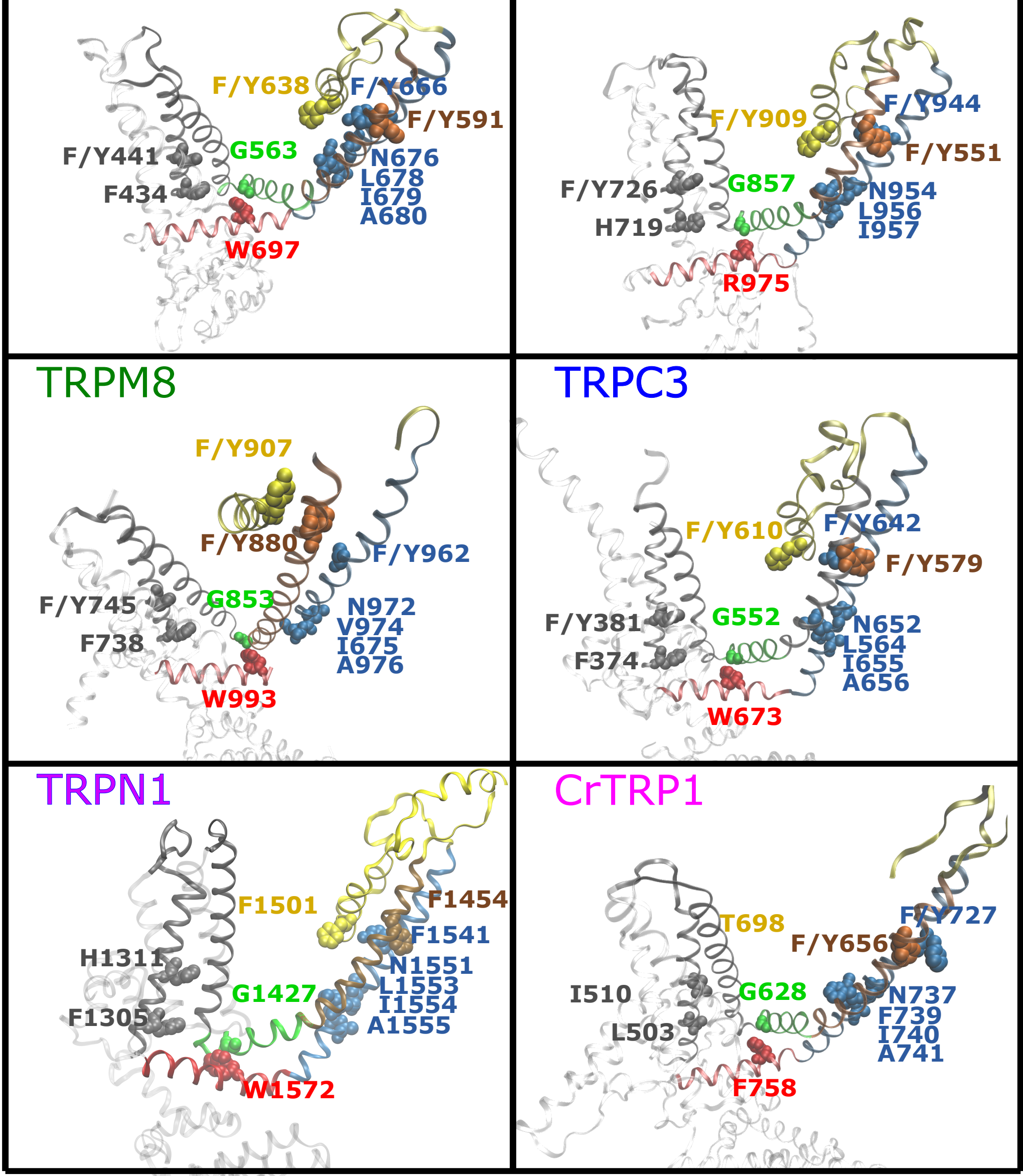

Figure S2. TRP fingerprint. Conserved residues and the forming patches are presented for a member of each TRP subfamily. Residues are colored according to the color code in Fig. 2 . Structures used: rTRPV1, PDB: 5IRZ; faTRPM8, PDB:6BPQ; hTRPC3, PDB:5ZBG; hTRPA1, PDB:3J9P; CrTRP1, PDB:6PW5. 


\section{TRPV1

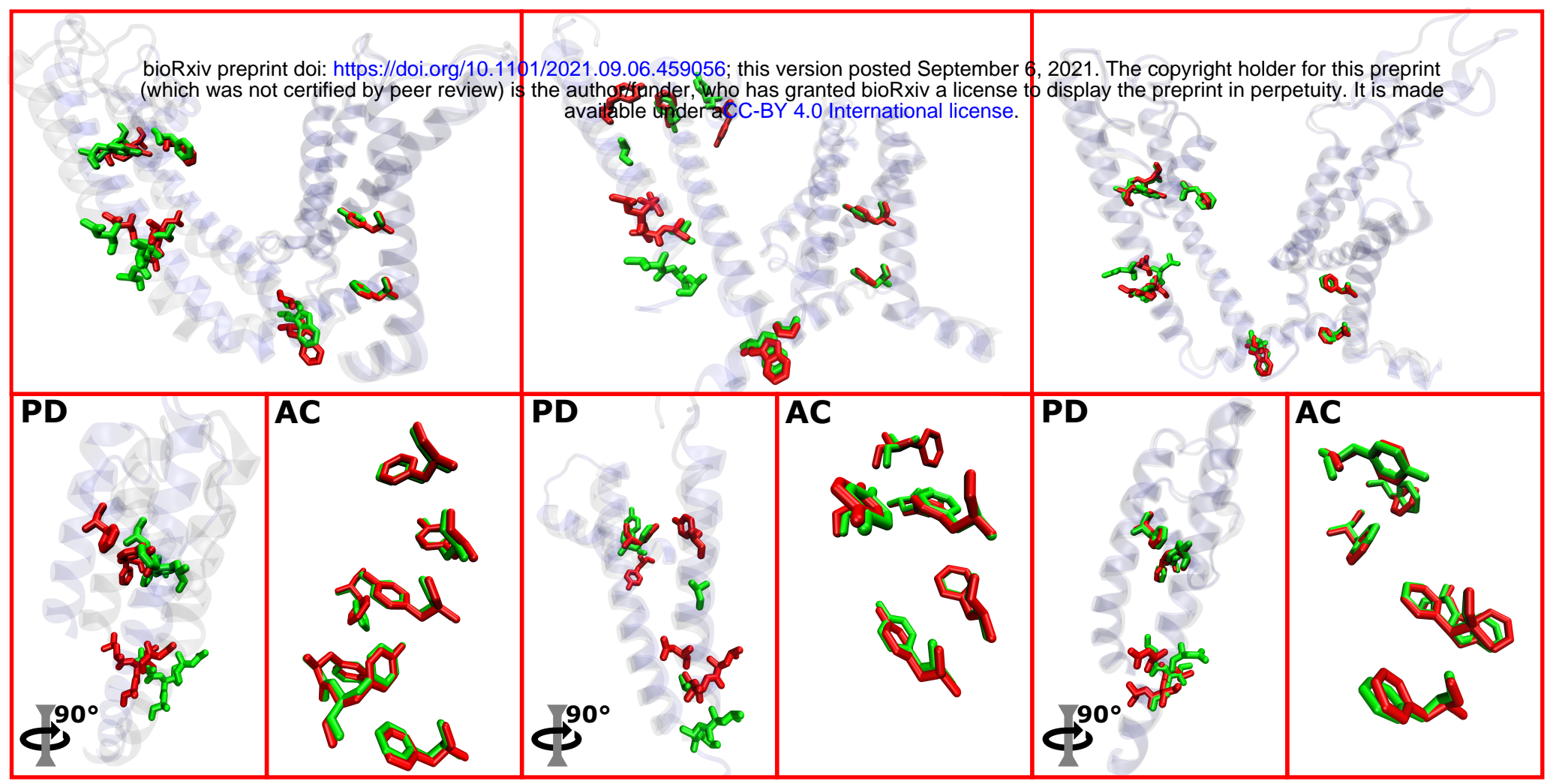

Figure S4. Conformation-dependent changes on spatial distribution of conserved residues Upper boxes: Relative position of fingerprint residues in the apo(green licorices on gray ribbons) and ligand bound (red licorices on blue ribbons) structures. Bottom boxes: Relative position of residues from the pore domain (PD) and the aromatic core (AC) comparing between apo and ligand bound structures. (rTRPV1 apo, PDB: 5IRZ; TRPV1ligand-bound, PDB: 5IRX; faTRPM8 apo, PDB: 6BPQ; faTRPM8 ligand-bound: 6NR2; hTRPC3 apo, PDB: 5ZBG; hTRPC3 ligand-bound, PDB: 6CUD). 


\section{a $\quad$ TRPV1}

bioRxiv preprint doi: http: ://doi.org/ 10.1101/2021.09.06.459056; this version posted September 6, 2021. The copyright holder for this preprint (which was not certified by peer review) is the author/funder, who has granted bioRxiv a license to display the preprint in perpetuity. It is made
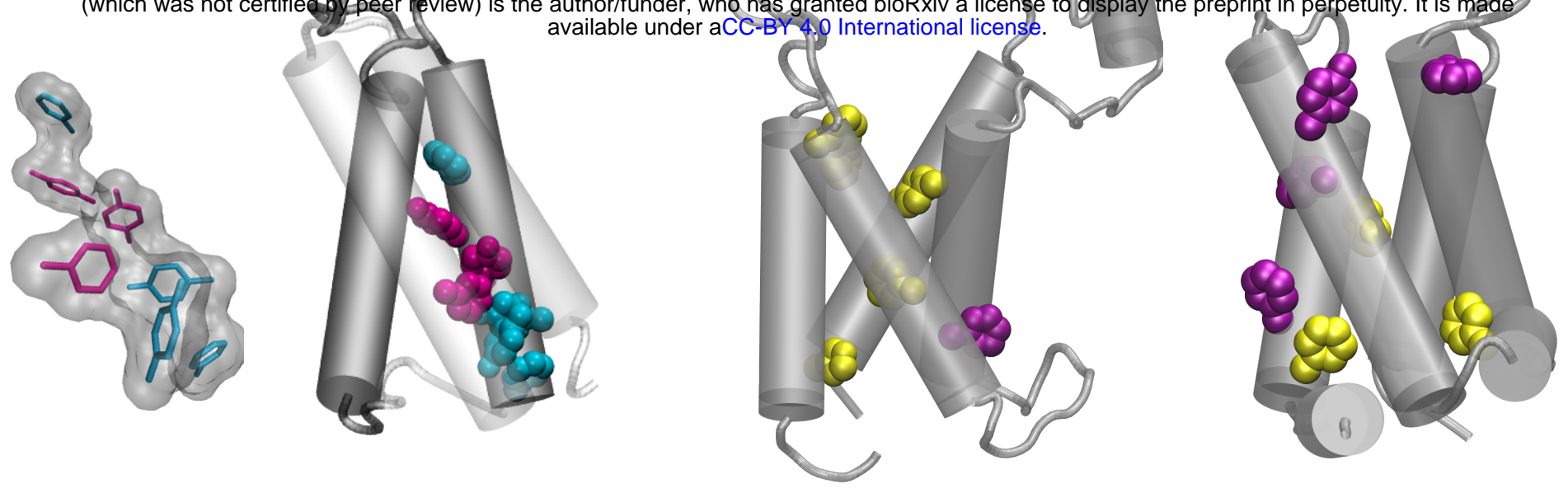

Figure S5. Aromatic residues in non-TRP channels. a) TRPV1 aromatic core. Cyan licorice/spheres: conserved residues ( $>50 \%$ ) in the specified subfamily, Magenta licorice/spheres: conserved residue ( $>50 \%)$ within subfamilies. b) aromatic residues found in the VSD of Kv1.2 and Nav1.4. Magenta: aromatic residues aligning with the conserved aromatic in at least one TRP subfamily. Yellow: non-aligned aromatic residues present in VSD. Structures: rTRPV1 apo, PDB: 5IRZ; rKv1.2, PDB:3LUT and hNav1.4, PDB:6AGF. 